\title{
Comparison of the ion-to-electron temperature ratio prescription: GRMHD simulations with electron thermodynamics
}

\author{
Yosuke Mizuno $^{1,2 \star}$, Christian M. Fromm ${ }^{3,2,4}$, Ziri Younsi ${ }^{5,2}$, Oliver Porth ${ }^{6}$, \\ Hector Olivares $^{7,2}$, Luciano Rezzolla ${ }^{2,8,9}$ \\ ${ }^{1}$ Tsung-Dao Lee Institute and School of Physics \& Astronomy, Shanghai Jiao-Tong University, Shanghai, 200240, People's Republic of China \\ ${ }^{2}$ Institut für Theoretische Physik, Goethe Universität, Max-von-Laue-Str. 1, 60438 Frankfurt am Main, Germany \\ ${ }^{3}$ Black Hole Initiative at Harvard University, 20 Garden Street, Cambridge, MA 02138, USA \\ ${ }^{4}$ Max-Planck-Institut für Radioastronomie, Auf dem Hügel 69, D-53121 Bonn, Germany \\ ${ }^{5}$ Mullard Space Science Laboratory, University College London, Holmbury St. Mary, Dorking, Surrey RH5 6NT, UK \\ ${ }^{6}$ Anton Pannekoek Institute for Astronomy, University of Amsterdam, Science Park 904, 1098 XH, Amsterdam, The Netherlands \\ ${ }^{7}$ Department of Astrophysics/IMAPP, Radboud University Nijmegen, P.O. Box 9010, 6500 GL Nijmegen, The Netherlands \\ ${ }^{8}$ Frankfurt Institute for Advanced Studies, Ruth-Moufang-Strasse 1, 60438 Frankfurt, Germany \\ ${ }^{9}$ School of Mathematics, Trinity College, Dublin 2, Ireland
}

Accepted XXX. Received YYY; in original form ZZZ

\begin{abstract}
The Event Horizon Telescope (EHT) collaboration, an Earth-size sub-millimetre radio interferometer, recently captured the first images of the central supermassive black hole in M87. These images were interpreted as gravitationally-lensed synchrotron emission from hot plasma orbiting around the black hole. In the accretion flows around low-luminosity active galactic nuclei such as M87, electrons and ions are not in thermal equilibrium. Therefore, the electron temperature, which is important for the thermal synchrotron radiation at EHT frequencies of $230 \mathrm{GHz}$, is not independently determined. In this work, we investigate the commonly used parameterised ion-to-electron temperature ratio prescription, the so-called $\mathrm{R}-\beta$ model, considering images at $230 \mathrm{GHz}$ by comparing with electron-heating prescriptions obtained from general-relativistic magnetohydrodynamical (GRMHD) simulations of magnetised accretion flows in a Magnetically Arrested Disc (MAD) regime with different recipes for the electron thermodynamics. When comparing images at $230 \mathrm{GHz}$, we find a very good match between images produced with the R- $\beta$ prescription and those produced with the turbulentand magnetic reconnection- heating prescriptions. Indeed, this match is on average even better than that obtained when comparing the set of images built with the $\mathrm{R}-\beta$ prescription with either a randomly chosen image or with a time-averaged one. From this comparative study of different physical aspects, which include the image, visibilities, broadband spectra, and light curves, we conclude that, within the context of images at $230 \mathrm{GHz}$ relative to MAD accretion flows around supermassive black holes, the commonly-used and simple $\mathrm{R}-\beta$ model is able to reproduce well the various and more complex electron-heating prescriptions considered here.
\end{abstract}

Key words: black hole physics - accretion, accretion discs - MHD - radiative transfer methods: numerical

\section{INTRODUCTION}

High-frequency very-long-baseline interferometry (VLBI) on Earth-sized baselines can resolve the immediate vicinity of nearby supermassive black hole (SMBH) event horizons. The Event Horizon Telescope (EHT) collaboration was established to build a global $1.3 \mathrm{~mm}$-wavelength VLBI network with the aim of capturing images of its primary targets: Sagittarius A* $\left(\mathrm{Sgr} \mathrm{A}^{*}\right)$, the SMBH at

^ E-mail: mizuno@sjtu.edu.cn (YM) the centre of our Galaxy, and Messier 87 (M87), the active galactic nucleus (AGN) at the heart of the Virgo A galaxy (Doeleman et al. 2008; Goddi et al. 2017). In April 2017, the EHT made the first observations with a full array capable of imaging with all eight participating radio telescopes, revealing an asymmetric ring morphology of the central compact radio source in M87 (Event Horizon Telescope Collaboration et al. 2019a,b,c,d,e,f). This image is interpreted as gravitationally-lensed emission surrounding the black hole shadow (Event Horizon Telescope Collaboration et al. 2019a,e).

The mass-accretion rates of M87 and Sgr A* are several or- 
ders of magnitude less than the Eddington limit and, hence, the corresponding luminosities of M87 and $\mathrm{Sgr} \mathrm{A}^{*}$ are significantly lower than their respective Eddington luminosities Ho (e.g., 2009); Prieto et al. (e.g., 2016). Furthermore, recent Faraday-rotation measurements of Sgr A* and M87 have provided indirect evidence of low mass-accretion rates Bower et al. (e.g., 2003); Marrone et al. (e.g., 2007); Kuo et al. (e.g., 2014). In this regime, material accreting onto the central black hole is understood to be in the radiatively inefficient accretion flow (RIAF) state, which comprises a geometrically thick and optically thin accretion disc (e.g., Narayan \& Yi 1994; Yuan \& Narayan 2014). RIAF models have been employed to investigate the innermost accretion flow structures for EHT target objects via semi-analytic approaches (e.g., Broderick \& Loeb 2006; Broderick et al. 2009, 2011, 2016; Pu et al. 2016; Pu \& Broderick 2018). In the past, many general-relativistic magnetohydrodynamical (GRMHD) simulations have been performed for single-fluid RIAFs onto rotating black holes for the study of event horizon-scale emission (e.g., Noble et al. 2007; Mościbrodzka et al. 2009, 2012, 2014, 2016; Dexter et al. 2009, 2010; Shcherbakov et al. 2012; Chan et al. 2015; Gold et al. 2017; Porth et al. 2017; Mizuno et al. 2018; Davelaar et al. 2018, 2019).

In hot and low-density accretion flows such as RIAFs, Coulomb coupling between electrons and ions is inefficient (e.g., Mahadevan \& Quataert 1997; Mahadevan 1998; Yuan \& Narayan 2014), and electrons and ions are not in thermal equilibrium. In most singlefluid MHD simulations, the ion temperature is dominant and therefore the electron temperature, which is important for the radiation, cannot be determined directly.

For modelling the emission from single-fluid GRMHD simulations, the ion-to-electron temperature ratio is typically set manually in radiation post-processing calculations. The simplest prescriptions for the electron temperature take $T_{\mathrm{i}} / T_{\mathrm{e}}$ to be constant (Mościbrodzka et al. 2009), dividing the simulation regions into jet and disc components, with different temperature ratios in each region (e.g., Mościbrodzka et al. 2014; Chan et al. 2015). Mościbrodzka et al. (2016) introduced a simple formula, the so-called "R- $\beta$ " prescription, which is associated with plasma magnetisation. This ion-to-electron temperature ratio prescription has been used in the development of theoretical model observations in the image library of M87 by the EHT (Event Horizon Telescope Collaboration et al. 2019e). Anantua et al. (2020) have proposed several new parameterised prescriptions as a function of the plasma beta or the magnetic pressure, which are termed: critical beta electron temperature model, constant electron beta model, and magnetic bias model. Typically, a high ion-to-electron temperature ratio implies that the electron heating does not impact the dynamics of the plasma flows because the electron pressure is low.

Recently, Ressler et al. (2015) presented a new formulation of GRMHD simulations which allows for the self-consistent evolution of the electron fluid, including, independent of one another, the effects of electron heating and conduction of heat flux along magnetic field lines. This approach is applied to the modelling of Sgr A* images and spectra (Ressler et al. 2017). Dexter et al. (2020) performed a parameter survey of Sgr A* using different black-hole spins, electron-heating prescriptions, and different accretion flow properties. Sądowski et al. (2017) and Ryan et al. (2017) have extended this formulation to include the effects of radiative feedback. This has been applied to investigate images and variabilities of Sgr A* (Chael et al. 2018) and M87 (Ryan et al. 2018; Chael et al. 2019).

From previous studies, we have two main approaches for the modelling of ion-to-electron temperature ratio from GRMHD sim- ulations: either setting the ratio manually in the post-processing calculation, or calculating the ratio from a more self-consistent evolution of the electron fluid from GRMHD simulations. However, so far, direct comparison between these two approaches has not been explored in detail. Consequently, in this work we seek to compare the simplified R- $\beta$ model using $230 \mathrm{GHz}$ EHT images, time variability at $230 \mathrm{GHz}$, and the corresponding broadband spectra with the results obtained from electron-heating prescriptions of GRMHD simulations of accretion flows onto a black hole with electron thermodynamics. In Sec. 2, we present our numerical approach and initial setup of GRMHD simulations and general-relativistic radiative transfer (GRRT) calculations.

In this study, we focus on one accretion scenario, the Magnetically Arrested Disc (MAD) (e.g., Narayan et al. 2003; Tchekhovskoy et al. 2011). We show our comparison results in $230 \mathrm{GHz}$ images, time variability of the $230 \mathrm{GHz}$ flux, and spectra from different black-hole spins, different electron-heating prescriptions, and different inclination angles in Sec. 3. In Sec. 4, we discuss our findings and the limitations of our approach. We conclude in Sec. 5 .

Throughout this paper, we adopt units where the speed of light, $c=1$, and the gravitational constant, $G=1$. Self-gravity arising from the gas is neglected. We absorb a factor of $\sqrt{4 \pi}$ into the definition of the magnetic field 4-vector, $b^{\mu}$.

\section{NUMERICAL SETUP}

We have performed a set of three-dimensional (3D) GRMHD simulations of magnetised tori in a black hole using the BHAC code (Porth et al. 2017; Olivares et al. 2019). Simulations are initialised with a Fishbone-Moncrief hydrodynamic equilibrium torus (Fishbone \& Moncrief 1976) with parameters $r_{\mathrm{in}}=20 r_{\mathrm{g}}$ and $r_{\max }=40 r_{\mathrm{g}}$, where $r_{\mathrm{g}} \equiv G M / c^{2}$ is the gravitational radius of the black hole and $M$ is its mass. An ideal-gas equation of state with a constant relativistic adiabatic index of $\Gamma_{\mathrm{g}}=4 / 3$ is used (Rezzolla \& Zanotti 2013). We note that some previous studies (Sądowski et al. 2017; Chael et al. 2018; Chael et al. 2019) have used a variable equation of state in which the adiabatic index depends on the temperature. This equilibrium torus solution is overlaid with a weak single magnetic field loop, whose radial distribution of the field profile is designed to supply enough magnetic flux onto the black hole to reach the magnetically arrested disc (MAD) state (e.g., Narayan et al. 2003; Tchekhovskoy et al. 2011). In order to excite the magneto-rotational instability (MRI) inside the torus, $1 \%$ of a random perturbation is applied to the gas pressure within the torus. In this paper, we choose three values for the dimensionless spin parameter: $a=-0.9375,0$, and 0.9375 .

The simulations are performed in spherical Modified KerrSchild coordinates. The outer radial boundary is located at $r=$ $2500 M$. The inner radial position of the simulation domain is well inside the black hole horizon in all cases. The grid resolution is $384 \times 192 \times 192$, including the full $2 \pi$ azimuthal domain. The simulations are evolved up to $t=15000 M$ in order to reach a quasi-steady state.

We solve the electron thermodynamics during the evolution of single-MHD fluid separately. For electron variables, we assume both charge neutrality and that the electron number density and four-velocity are the same as those of the ions, i.e., $n_{\mathrm{e}}=n_{\mathrm{i}}=n$ and $u_{\mathrm{e}}^{\mu}=u_{\mathrm{i}}^{\mu}=u^{\mu}$. However, the electron entropy equation is solved for the electron temperature separately:

$\rho T_{\mathrm{e}} \partial_{\mu} s_{\mathrm{e}}=f_{\mathrm{e}} Q$ 
where $\rho$ is the fluid rest-mass density, $s_{\mathrm{e}}=\left(\Gamma_{\mathrm{e}}-1\right)^{-1} \log \left(p_{\mathrm{e}} / \rho^{\Gamma_{\mathrm{e}}}\right)$ is electron entropy, $\Gamma_{\mathrm{e}}$ is adiabatic index for the electrons, $p_{\mathrm{e}}$ is the electron pressure, $f_{\mathrm{e}}$ is a fraction of the dissipative heating which goes into electrons and $Q$ is the total heating rate per unit volume. Here we neglect the energy exchange rate due to Coulomb coupling, anisotropic thermal heat flux, and radiative cooling which have been considered in previous works (Ressler et al. 2015, 2017; Chael et al. 2018; Chael et al. 2019). ${ }^{1}$ The total heating rate, $Q$, is calculated through direct comparison between the internal energy obtained from solving an electron entropy conservation equation and the total internal energy of gas as described in Ressler et al. (2015).

In order to apply the electron entropy equation in GRMHD simulations using the BHAC code, eq. (1) has been rewritten as

$\partial_{\mu}\left(\sqrt{-g} \rho u^{\mu} \kappa_{\mathrm{e}}\right)=\frac{\sqrt{-g}\left(\Gamma_{\mathrm{e}}-1\right)}{\rho^{\Gamma_{\mathrm{e}}-1}} f_{\mathrm{e}} Q$,

where $\kappa_{\mathrm{e}} \equiv \exp \left[\left(\Gamma_{\mathrm{e}}-1\right) s_{\mathrm{e}}\right]$. Without the heat conduction term, equation (2) takes a conservative form, with conserved quantities $U_{\kappa_{\mathrm{e}}} \equiv \sqrt{-g} \rho u^{t} \kappa_{\mathrm{e}}$ and flux $F_{\kappa_{\mathrm{e}}}^{i} \equiv \sqrt{-g} \rho u^{i} \kappa_{\mathrm{e}}$. In order to obtain the time evolution of $\kappa_{\mathrm{e}}$, we use operator splitting by following Ressler et al. (2015): (i) solve the conservative equation without a source term, $S_{\kappa_{\mathrm{e}}}$, (ii) update $\kappa_{\mathrm{e}}$ with the heating in the source term explicitly.

For the evaluation of heating in each time step, the entropy conversation equation $\left(U_{\kappa_{\mathrm{g}}} \equiv \sqrt{-g} \rho u^{t} \kappa_{\mathrm{g}}, F_{\kappa_{\mathrm{g}}}^{i} \equiv \sqrt{-g} \rho u^{i} \kappa_{\mathrm{g}}\right.$, and $S_{K_{\mathrm{g}}}=0$ ) is introduced as a reference to compare with the energy conservation equation. The heating update to the electrons is calculated by the difference between the entropy obtained from the total energy conservation equation $\left(\kappa_{\mathrm{g}}\right)$ and the entropy obtained from the entropy conservation equation $\left(\hat{\kappa}_{\mathrm{g}}\right)$ as given by

$\kappa_{\mathrm{e}}^{n+1}=\hat{\kappa}_{\mathrm{e}}^{n+1}+\frac{\Gamma_{\mathrm{e}}-1}{\Gamma_{\mathrm{g}}-1}\left(\rho^{\Gamma_{\mathrm{g}}-\Gamma_{\mathrm{e}}} f_{\mathrm{e}}\right)^{n+1 / 2}\left(\kappa_{\mathrm{g}}-\hat{\kappa_{\mathrm{g}}}\right)^{n+1}$,

where $\hat{\kappa}_{\mathrm{e}}$ is the solution from the electron entropy conservation equation without a source term.

In GRMHD simulations, the heating is provided by grid-scale dissipation which is related to magnetic reconnection, shock heating, Ohmic heating, and turbulent heating. In this work, we consider two heating prescriptions: turbulent heating and magnetic reconnection, to determine the heating fraction $f_{e}$. In the turbulent heating model, we use the results of numerical simulations of damping of MHD turbulence by Kawazura et al. (2019):

$f_{\mathrm{e}}=\frac{1}{1+Q_{\mathrm{i}} / Q_{\mathrm{e}}}$,

where

$\frac{Q_{\mathrm{i}}}{Q_{\mathrm{e}}}=\frac{35}{1+(\beta / 15)^{-1.4} \exp \left(-0.1 T_{\mathrm{e}} / T_{\mathrm{i}}\right)}$,

and where $\beta \equiv p_{\mathrm{g}} / p_{\mathrm{m}}$ is the plasma-beta parameter, the ratio between the fluid pressure and magnetic pressure $p_{\mathrm{m}}=b^{2} / 2$. This formula expresses a transition with increasing $\beta$ from an electrondominated heating to a proton-dominated one; this transition takes place around the value of $\beta \sim 1$. Howes (2010) has provided fitting

1 Ressler et al. (2015) have included anisotropic conduction of heat along magnetic field lines. They reported that this conduction has little effect on the spectrum and image. Chael et al. (2018) have considered radiative cooling of electrons and Coulomb coupling of electrons to ions. These effects are mostly unimportant for low accretion rate systems such as Sgr A* and M87. formulae derived from the linear theory of damping of MHD turbulence, showing quantitatively similar behaviour to that described by Kawazura et al. (2019). Recently, Kawazura et al. (2020) extended their investigation and provided new fitting formulae of the ion-toelectron heating rate by turbulence, including the compressive-toAlfvénic driving power ratio. In this work, we have not adopted this formula for turbulent heating model. In the magnetic reconnection model, we employ a fitting function as measured in particle-in-cell simulations of magnetic reconnection described by Rowan et al. (2017):

$f_{\mathrm{e}}=\frac{1}{2} \exp \left[\frac{-\left(1-\beta / \beta_{\max }\right)}{0.8+\sigma_{\mathrm{h}}^{0.5}}\right]$,

where $\beta_{\max }=\sigma_{\mathrm{h}} / 4, \sigma_{\mathrm{h}}=b^{2} / \rho h$ is magnetisation as defined with respect to the fluid specific enthalpy $h=1+\Gamma_{\mathrm{g}} p_{\mathrm{g}} /\left(\Gamma_{\mathrm{g}}-1\right)$. In the formula for the magnetic-reconnection heating prescription, $f_{\mathrm{e}}$ reaches its maximum value, i.e., $1 / 2$, in highly magnetised regions $\left(\sigma_{\mathrm{h}} \gg 1\right)$ or in regions with large $\beta$. On the other hand, in regions with small $\beta$ ( $\left.\beta \ll \beta_{\max }\right), f_{\mathrm{e}}$ attains small values that depend on $\sigma_{\mathrm{h}}$. Finally, in the limit of non-relativistic reconnection, i.e., $\sigma_{\mathrm{h}} \ll 0.1, f_{\mathrm{e}} \rightarrow 0.14$. Recently Rowan et al. (2019) has provided a new fitting formula for the heating prescription for the magnetic reconnection model, including the effect of guide fields. A quantitatively similar behaviour to that found in Rowan et al. (2017) was demonstrated. We here perform GRMHD simulations considering two different electron heating prescription - either turbulent or magnetic-reconnection heating - and three different black-hole spins, for a total six 3D GRMHD simulations.

In the GRMHD simulations, we take the adiabatic index of the electron fluid to be $\Gamma_{\mathrm{e}}=4 / 3$ and initially set the electron internal energy density $u_{\mathrm{e}}$ to $10 \%$ of the fluid internal energy density $u_{\mathrm{g}}$. The evaluation of the implementation of the electron-heating calculations in the BHAC code and its convergence is presented in Appendix A, where we show its correct implementation and convergence when $\Gamma_{\mathrm{g}}=\Gamma_{\mathrm{e}}$.

As customary in codes solving the equations of relativistic hydrodynamics or those of GRMHD, the occurrence of vacuum is avoided by introducing a very low-density fluid, i.e., an atmosphere, filling regions that are far from the high-density fluid (Rezzolla \& Zanotti 2013). In essence, floor values are applied to the rest-mass density, $\rho_{\mathrm{fl}}=10^{-4} r^{-2}$ and the gas pressure, $p_{\mathrm{fl}}=\left(10^{-6} / 3\right) r^{-2 \Gamma_{\mathrm{g}}}$, so that in all the numerical cells for which $\rho \leq \rho_{\mathrm{fl}}$ or $p \leq p_{\mathrm{fl}}$, we simply set $\rho=\rho_{\mathrm{fl}}$ and $p=p_{\mathrm{fl}}$. Similarly, a ceiling is introduced in those regions of high magnetisation, so that we set $\sigma_{\max }=100$ in all those cells for which $\sigma \geq \sigma_{\max }$. Finally, for the electron entropy we apply both a floor and a ceiling to the electron pressure. More specifically, if the electron pressure is less than $1 \%$ of the floor value of the gas pressure $p_{\mathrm{fl}}$, we then reset $p_{\mathrm{e}}=0.01 p_{\mathrm{fl}}$. At the same time, if the electron pressure is larger than the gas pressure, we reset $p_{\mathrm{e}}=0.99 p_{\mathrm{g}}$. We note that we re-calculate the gas entropy $\kappa_{\mathrm{g}}$ and the electron entropy $\kappa_{\mathrm{e}}$ in those cells where the density is floored.

In order to obtain the synthetic image from GRMHD simulations, we perform GRRT calculations in post-processing using BHOSS (Younsi et al. 2012, 2020). The BHOSS code performs GRRT calculations of the GRMHD simulation data in post-processing. The equations of GRRT are solved along geodesics integrated through this GRMHD data, and the resultant images, light curves and spectra as seen by a distant observer for a given viewing angle and observing frequency are determined.

In this work, we adopt a relativistic thermal Maxwell-Jünttner 
electron distribution function for the synchrotron absorption and emission given by Leung et al. (2011). For the electron-ion temperature ratio, we consider two different approaches. The first uses the aforementioned $\mathrm{R}-\beta$ prescription:

$\frac{T_{\mathrm{i}}}{T_{\mathrm{e}}}=\frac{1}{1+\beta^{2}} R_{\mathrm{l}}+\frac{\beta^{2}}{1+\beta^{2}} R_{\mathrm{h}}$.

In the $\mathrm{R}-\beta$ prescription, the temperature ratio in the strongly magnetised regions $\left(\beta_{p} \ll 1\right)$ like the jet funnel is $T_{\mathrm{i}} / T_{\mathrm{e}} \sim R_{\mathrm{l}}$ and in weakly magnetised regions $\left(\beta_{p} \ll 1\right)$, such as the disc, this tends to $T_{\mathrm{i}} / T_{\mathrm{e}} \sim R_{\mathrm{h}}$. In our work, we fix $R_{\mathrm{l}}=1$ and vary $R_{\mathrm{h}}$ as $R_{\mathrm{h}}=1$, $5,10,20,40,80$, and 160, a similar choice to that of Event Horizon Telescope Collaboration et al. (2019e). Here, we keep $R_{1}=1$ fixed and vary $R_{\mathrm{h}}$ from 1 to 160 , following the analysis carried out by Event Horizon Telescope Collaboration et al. (2019e). Nevertheless, in Appendix $\mathrm{C}$, we investigate the effect of adopting different values for $R_{1}$ and conclude that the image-comparison results do not depend on the choice made for $R_{1}$.

The second approach takes the ion-to-electron temperature ratio directly from the electron-heating prescription calculated in the GRMHD simulations. The electron pressure is computed as $p_{\mathrm{e}}=\kappa_{\mathrm{e}} \rho^{\Gamma_{\mathrm{e}}}$, where the values of $\kappa_{\mathrm{e}}$ are updated as in Eq. (3). The ion-to-electron temperature ratio is then calculated as $T_{\mathrm{i}} / T_{\mathrm{e}}=\left(p_{\mathrm{g}}-p_{\mathrm{e}}\right) / p_{\mathrm{e}}$.

The dimensionless electron temperature is then given by:

$\Theta_{\mathrm{e}}=\left(\frac{p_{\mathrm{g}}-p_{\mathrm{e}}}{\rho}\right)\left(\frac{m_{\mathrm{p}} / m_{\mathrm{e}}}{T_{\mathrm{i}} / T_{\mathrm{e}}}\right)$,

where $m_{\mathrm{p}}$ and $m_{\mathrm{e}}$ are the proton and electron masses, respectively, and we assume a fully ionised hydrogen plasma. ${ }^{2}$ The electron temperature in c.g.s. units is calculated as $T_{\mathrm{e}}=m_{\mathrm{e}} c^{2} \Theta_{\mathrm{e}} / k_{\mathrm{B}}$, where $k_{\mathrm{B}}$ is the Boltzmann constant.

Although GRMHD simulations are scale-free, the GRRT calculation depends on the physical mass scale. Here we consider our target central black hole to be M87 with a mass $M=6.5 \times 10^{9} M_{\odot}$ at a distance $D=16.8 \mathrm{Mpc}$ (Event Horizon Telescope Collaboration et al. 2019f). We set the mass scale unit which is the conversion from simulation density to physical density by normalising the time-averaged flux at $230 \mathrm{GHz}$ to the value of $0.5 \mathrm{Jy}$ (Event Horizon Telescope Collaboration et al. 2019e).

In GRRT calculations, we limit the emission regions in which we can reasonably trust the fluid thermodynamics by imposing a threshold on the magnetisation $\sigma=b^{2} / \rho$. We only consider emission that originates from the regions where $\sigma<1$. In regions with $\sigma>1$, the fluid density and temperature may be affected by the simulation floors and are therefore unreliable. Furthermore, we also adopt the 'fast light' approximation. In this approximation, we calculate a emission on a fixed time slice of fluid quantities which means the light propagation time across the domain is small compared to the dynamical timescale of the system.

\section{RESULTS}

\subsection{GRMHD Simulations with Electron Thermodynamics}

In order to investigate the properties of accretion flows quantitatively, we calculate the volume-integrated mass-accretion rate and

\footnotetext{
2 In the R- $\beta$ parameterisation, we use $p_{\mathrm{g}}$ instead of $p_{\mathrm{g}}-p_{\mathrm{e}}$ because it is not possible to estimate $p_{\mathrm{e}}$ from one-temperature GRMHD simulations. This is equivalent to assuming $T_{\mathrm{i}} \simeq T_{\mathrm{g}}$ and $T_{\mathrm{i}} \gg T_{\mathrm{e}}$ (e.g., Mościbrodzka et al. 2016).
}
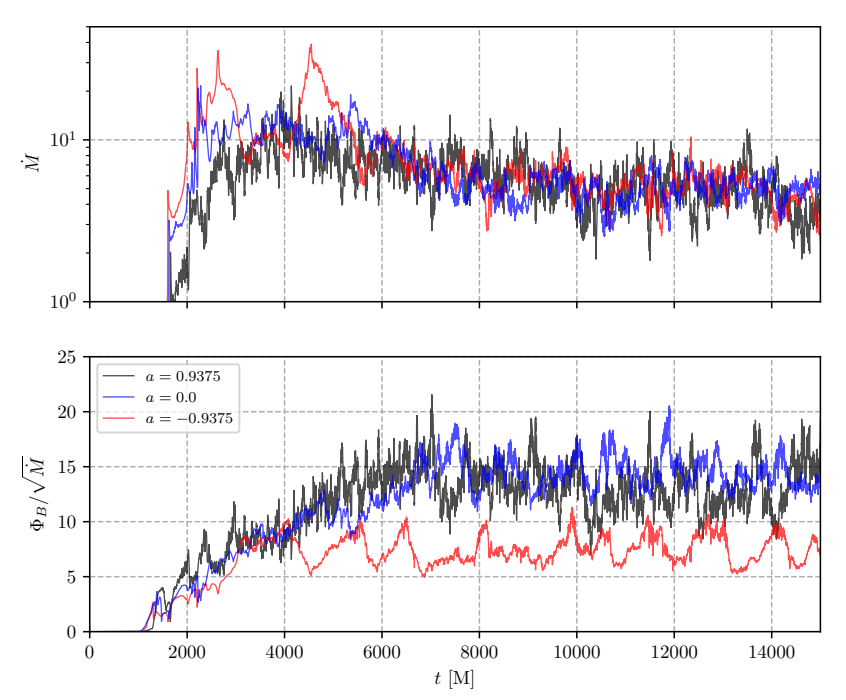

Figure 1. (Top) mass-accretion rate and (bottom) magnetic flux rate at the black hole horizon for a black hole with $a=-0.9375$ (red), 0 (blue), and 0.9375 (black).

magnetic flux rate at the black hole horizon. Following Porth et al. (2019) we define the mass-accretion rate as

$\dot{M}=\int_{0}^{2 \pi} \int_{0}^{\pi} \rho u^{r} \sqrt{-g} d \theta d \phi$,

while the magnetic flux rate is written as

$\Phi_{\mathrm{B}}=\frac{1}{2} \int_{0}^{2 \pi} \int_{0}^{\pi}\left|B^{r}\right| \sqrt{-g} d \theta d \phi$.

Figure 1 shows time evolution of mass-accretion rate $(\dot{M})$ and dimensionless magnetic flux rate at the black hole horizon $\left(\dot{\phi_{\mathrm{B}}} / \sqrt{\dot{M}}\right)$ in black-hole spin cases with $a=-0.9375$ (red), 0 (blue), and 0.9375 (black). The mass-accretion rates have very similar profiles amongst different black-hole spin cases, which gradually decrease with time after $t=4000 \mathrm{M}$. The dimensionless magnetic flux saturates at the maximum values of $\Phi_{\mathrm{B}} / \sqrt{\dot{M}} \simeq 15$ in the cases with $a=0.9375$ and 0 . In counter-rotating case with $a=-0.9375$, the maximum values of dimensionless magnetic flux adopts a somehow lower value of 8 . These values are consistent with Tchekhovskoy \& McKinney (2012). ${ }^{3}$ All cases reach the MAD state after $t=6000 \mathrm{M}$. As first discussed by Tchekhovskoy \& McKinney (2012), a counter-rotating accretion flow leads generically to a smaller saturation value of the magnetic flux than in the case of a co-rotating flow. Furthermore, the results obtained here for the magnetic flux are systematically smaller than those reported by Dexter et al. (2020) for co-rotating flows, hinting that the actual saturation value may depend on the initial disc thickness, which is different from the one adopted by Dexter et al. (2020).

Azimuthal- and time- averaged dimensionless electron temperatures $\left(\Theta_{e}\right)$ are shown in Figure 2. In the funnel wall region between the highly magnetised polar funnel and bound disc material, electrons are efficiently heated in both heating prescriptions, resulting

\footnotetext{
3 In our system of units, the dimensionless magnetic flux rate at the black hole horizon differs from the used definition in Tchekhovskoy et al. (2011); Tchekhovskoy \& McKinney (2012); McKinney et al. (2012) by a factor of $\sqrt{4 \pi}$.
} 

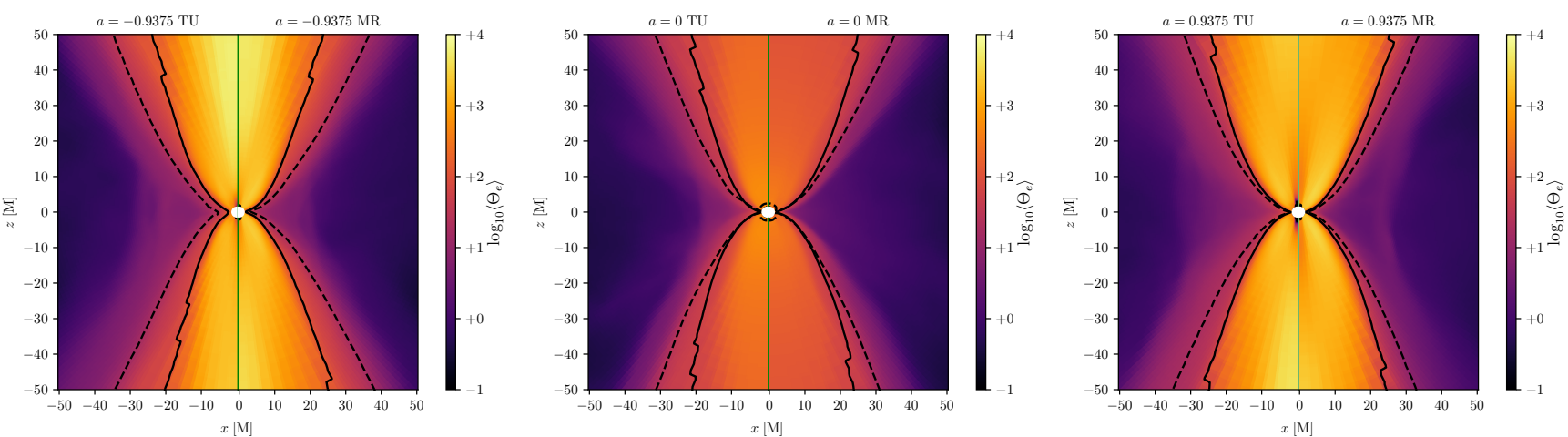

Figure 2. Azimuthal and time averaged dimensionless electron temperature $\Theta_{e}$ for black-hole spins with $a=-0.9375$ (left), 0 (middle), and 0.9375 (right) using the turbulent heating prescription (left side of the panels) and the magnetic-reconnection heating prescription (right side of the panels). The averaging is performed over the time interval $t=14000-15000 M$, which is when the simulations have reached a quasi-steady state. The black solid and dotted lines indicate $\sigma=1$ and Bernoulli parameter $-h u_{t}=1.02$, respectively.
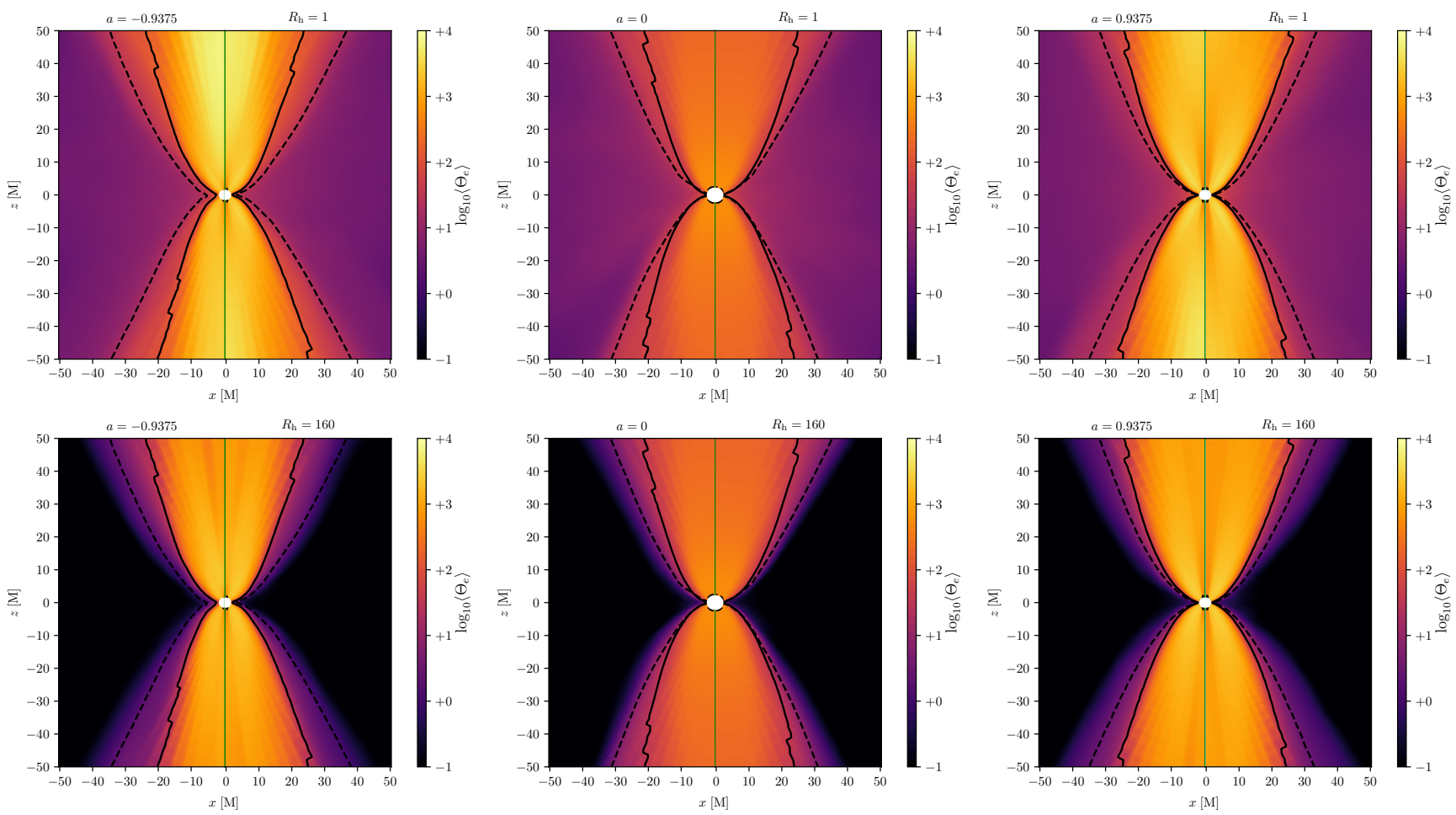

Figure 3. Same as Fig.2, but when using $R_{\mathrm{h}}=1$ (upper) and $R_{\mathrm{h}}=160$ (lower) in the R- $\beta$ parameterised prescription.

in a high ion-to-electron temperature ratio. This trend is similarly seen in Chael et al. (2019) and Dexter et al. (2020), which also used MAD simulations. We note that our MAD simulations exhibit efficient heating in the polar and funnel wall regions. This is due to the difference in the assumed adiabatic index of the fluid between GRMHD simulations. In our simulations, we assume an identical adiabatic index between fluid and electrons, i.e., $\Gamma_{\mathrm{g}}=\Gamma_{\mathrm{e}}=4 / 3$. In this case, electron heating occurs more efficiently via dissipation (see Appendix A). In our simulations, we neglect radiative cooling. As a consequence, this may lead to an overestimate in cases of high electron temperature.
As reported by Dexter et al. (2020), we also see a mild dependence on black-hole spin in the electron temperature, which becomes larger for higher black-hole spins. However, both the ion and the electron temperatures increase, so that the ion-to-electron temperature ratio does not vary appreciably. As a reference, we present the azimuthal- and time- averaged dimensionless electron temperature by using the $\mathrm{R}-\beta$ prescription with $R_{\mathrm{h}}=1$ and $R_{\mathrm{h}}=160$ in Fig. 3. The dimensionless electron temperature in the disc region becomes cooler for higher values of $R_{\mathrm{h}}$.

We note that the region near the poles is highly magnetised and may be affected by the numerical floor treatment. Therefore the ion- 


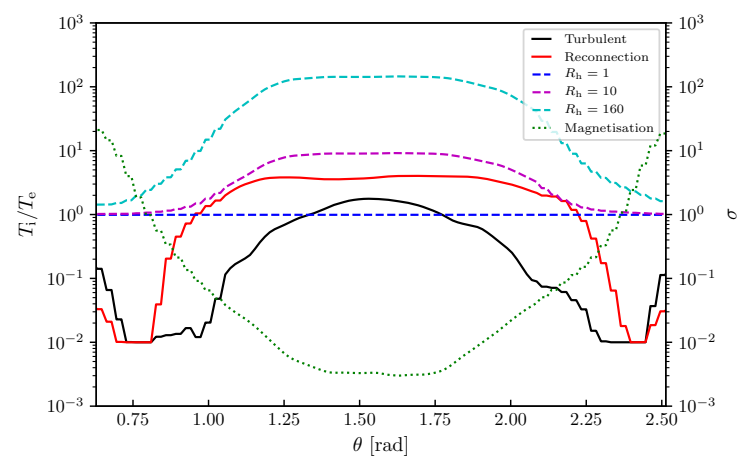

Figure 4. Azimuthal and time-averaged polar-angle profiles of the ion-toelectron temperature ratio of the MAD-accretion case with $a=0.9375$ at $r=$ $20 M$. Solid lines indicate turbulent (black) and magnetic reconnection (red) heating prescription. Dashed lines present $\mathrm{R}-\beta$ parameterised prescription with different $R_{\mathrm{h}}$ value, $R_{\mathrm{h}}=1$ (blue), 10 (magenta), and 160 (cyan). The green dotted line shows the magnetisation.

to-electron temperature ratio may be unreliable in this region and we omit the contribution of these regions in the GRRT calculations of images at $230 \mathrm{GHz}$ (the threshold is set with $\sigma \geq 1$ ).

Figure 4 presents azimuthal- and time- averaged polar-angle profiles of the ion-to-electron temperature ratio of the MAD model with $a=0.9375$ on a polar slice at $r=20 M$. Both the R- $\beta$ (dashed lines) and the electron-heating prescriptions (solid lines) produce a high ion-to-electron temperature ratio around the equatorial plane, although the R- $\beta$ generally yield larger temperatures than the reconnection-heating or the turbulent-heating prescriptions; similar plots have been shown by Chael et al. (2018). We note that the reconnection-heating prescription employed by Chael et al. (2018) leads to $T_{\mathrm{e}}<T_{\mathrm{i}}$ everywhere along the polar section, while in our calculations this is true only in the highly magnetised region. Because in this region radiative cooling would be effective, the electron heating computed in our simulations may be overestimated. Also, it should be noted that around the equatorial plane, both electron-heating prescription have a profile similar to that of the $\mathrm{R}-\beta$ prescriptions. Large differences are seen around the funnel region, where most of the electron heating takes place, so that $T_{\mathrm{i}} \ll T_{\mathrm{e}}$ there for both electron-heating prescriptions.

\subsection{GRRT Calculations}

In order to compare radiative signatures using different ionto-electron temperature ratio prescriptions, we have calculated $230 \mathrm{GHz}$ images using the GRRT code BHOSS (Younsi et al. 2012, 2020). Figures 5 and 6 show the time-averaged GRRT images at $i=163^{\circ}$ of MAD simulations with different black-hole spins: $a=-0.9375$ (top panels), 0 (middle panels), and 0.9375 (bottom panels) using the turbulent heating prescription, the magneticreconnection heating prescription, and the $\mathrm{R}-\beta$ model with $R_{\mathrm{h}}=1$ and $R_{\mathrm{h}}=160$, in a linear and logarithmic scale, respectively. Images are averaged from $t=14000 \mathrm{M}$ to $15000 \mathrm{M}$. All averaged images have the same total flux of $0.5 \mathrm{Jy}$. The camera field of view is set to be $640 \mu$ as $\times 640 \mu$ as, which is a four times larger field-of-view than the one used by the Event Horizon Telescope Collaboration et al. (2019e). For the black hole in M87, $100 r_{\mathrm{g}}$ corresponds to 382 $\mu$ as. Our choice of field of view corresponds to $167 r_{\mathrm{g}}$. We rotate the image to $252^{\circ}\left(72^{\circ}\right)$ North-to-East in co-rotating case (counterrotating case) to set the bright spot position to be in the South. In the co-rotating case, the approaching jet orientation is in a similar direction to the large scale jet (e.g., Hada et al. 2017; Kim et al. 2018; Walker et al. 2018).

In the time-averaged images, we effectively suppress the timedependent turbulent features of the individual GRRT images, thus allowing for more generic and persistent features of the GRRT images to emerge. In high black-hole spin cases, the asymmetry of the brightness distribution of the bright photon ring becomes stronger than the non-rotating black hole case, which is almost uniform. GRRT images at $230 \mathrm{GHz}$ in different ion-to-electron temperature ratio prescriptions are morphologically very similar, i.e., bright photon ring emission with some faint extended emission around it. However, the brightness distribution of the photon ring differs with the chosen heating prescriptions. In the turbulent heating prescription, the brightness distribution of the photon ring is more uniform than the magnetic reconnection heating prescription. A similar trend is seen for higher $R_{\mathrm{h}}$ values in the $\mathrm{R}-\beta$ model. In the images with a logarithmic scale, these trends are more clear. Both turbulent and magnetic heating prescriptions have more extended diffused emission than the $\mathrm{R}-\beta$ models. This is because both electron-heating prescriptions have higher ion-to-electron temperature ratios at the funnel wall region. In the turbulent heating prescription model, the maximum flux at $230 \mathrm{GHz}$ is lower than other models due to contributions from large extended diffused emission regions. This leads to a region representing the $1 \%$ level of the maximum flux (white dashed contour in Fig. 6) that is a little larger than in the $R-\beta$ models. On the other hand, the magnetic-reconnection heating prescription model has a higher maximum flux even though it has a more diffused extended emission region, which may not contribute much to the total flux. This is indicated by the position of the $1 \%$ level of maximum flux.

Figures 7 and 8 show the time-averaged GRRT images of MAD simulations at $i=60^{\circ}$ with different black-hole spins in different ion-to-electron temperature ratio prescriptions, in linear and logarithmic scales, respectively. At $i=60^{\circ}$, the asymmetry of the bright photon ring is more prominent than the $i=163^{\circ}$ case due to stronger Doppler beaming by the emitting plasma. The general trends of different ion-to-electron ratio prescriptions are unchanged. Images of turbulent heating prescriptions have a more uniform distribution over the bright photon ring than other models with lower maximum fluxes. In the logarithmic scale images, both turbulent heating and magnetic-reconnection heating prescriptions have a little widely extended diffused emission regions than the $\mathrm{R}-\beta$ prescription, even though the $\mathrm{R}-\beta$ prescription at $i=60^{\circ}$ has a more extended emission region than $i=163^{\circ}$. Considering the threshold of the $1 \%$ of the maximum flux, this region is marginally larger for the electron-heating model.

\subsection{Image Comparisons}

In order to provide a more quantitative comparison between electron-heating prescription models and R- $\beta$ models, we computed three image-comparison metrics: the mean square error (MSE), structural dissimilarity (DSSIM) (Wang et al. 2004), and difference of normalised cross-correlation coefficient (NCCC) from 1 (see e.g., Mizuno et al. 2018; Fromm et al. 2020). The MSE is a pixelby-pixel comparison metric calculated by averaging the squared intensity difference between two image pixels, namely

MSE $:=\frac{\sum_{j=1}^{N}\left|I_{j}-K_{j}\right|^{2}}{\sum_{j=1}^{N}\left|I_{j}\right|^{2}}$, 


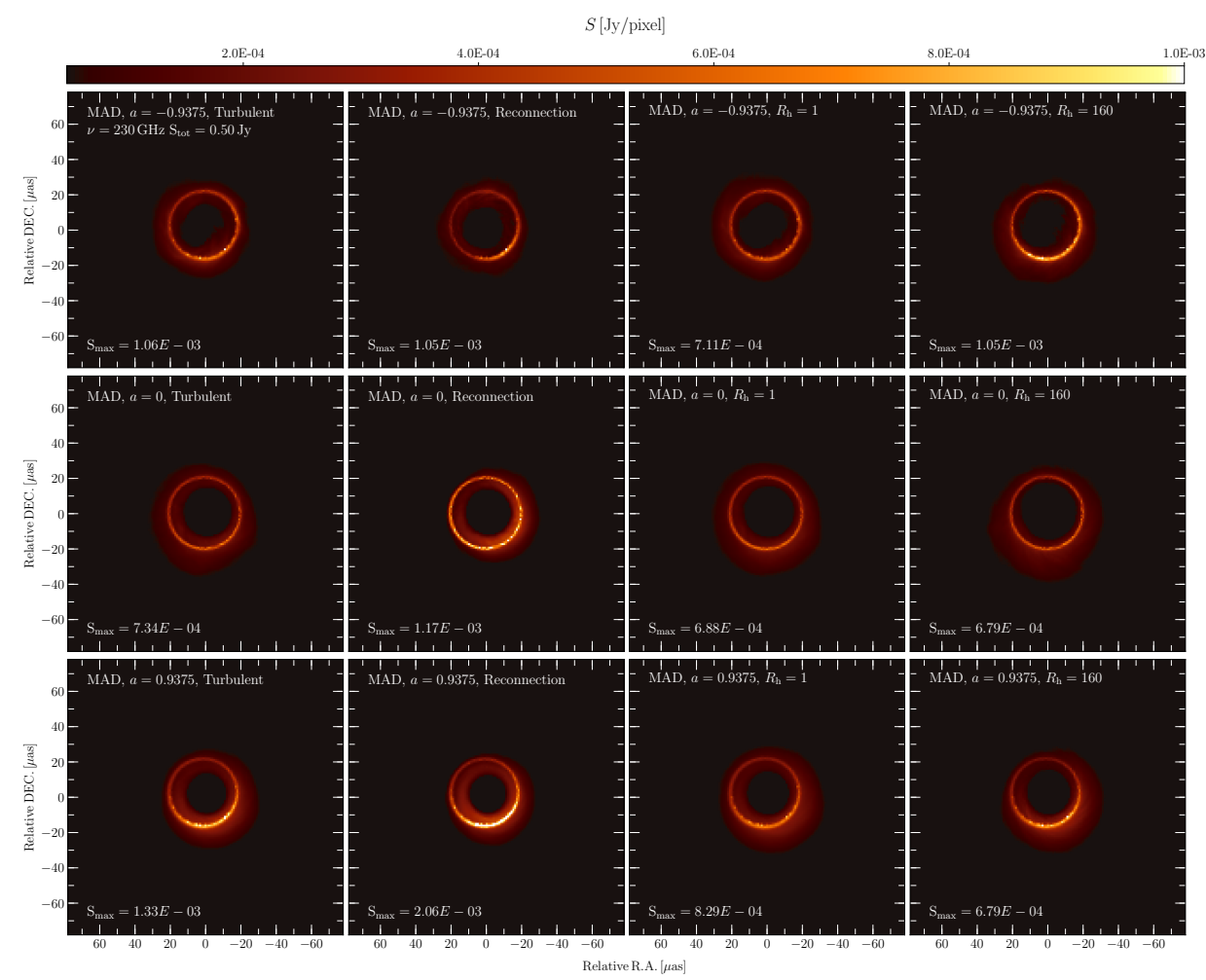

Figure 5. Time-averaged GRRT images at $i=163^{\circ}$ of MAD simulations with different black-hole spin cases, $a=-0.9375$ (top panels), 0 (middle panels), and 0.9375 (top panels). From left to right: images using turbulent heating prescription, magnetic-reconnection heating prescription, the R- $\beta$ model with $R_{\mathrm{h}}=1$, and $\mathrm{R}-\beta$ model with $R_{\mathrm{h}}=160$. The image is averaged with GRRT images from $t=14000 M$ to $15000 M$. All averaged images have the same total flux with $0.5 \mathrm{Jy}$ at $230 \mathrm{GHz}$.

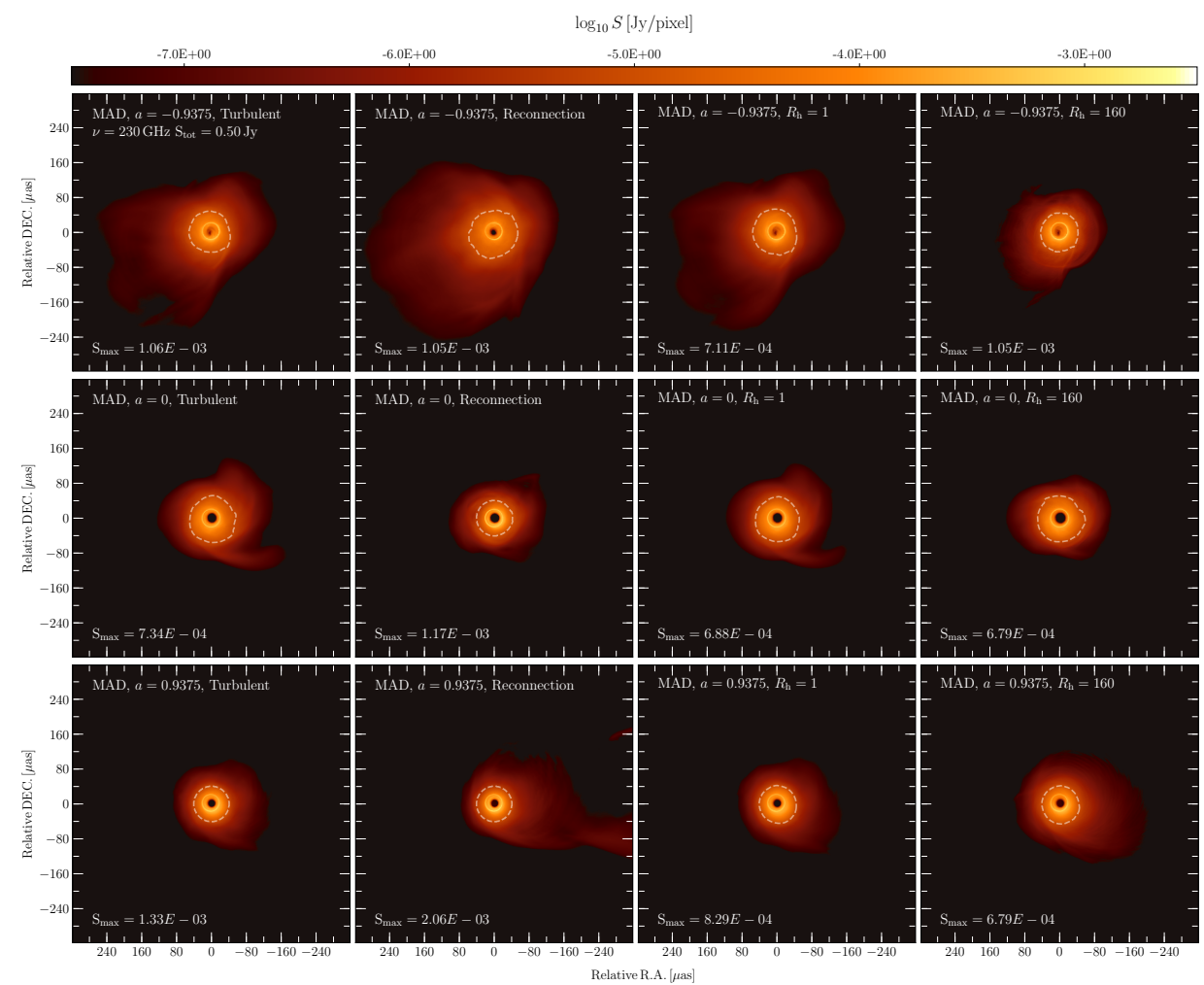

Figure 6. Same as Fig. 5, but plotting logarithmic scale. Dashed lines are indicated the 1\% of maximum intensity in each images. 
$S$ [Jy/pixel]

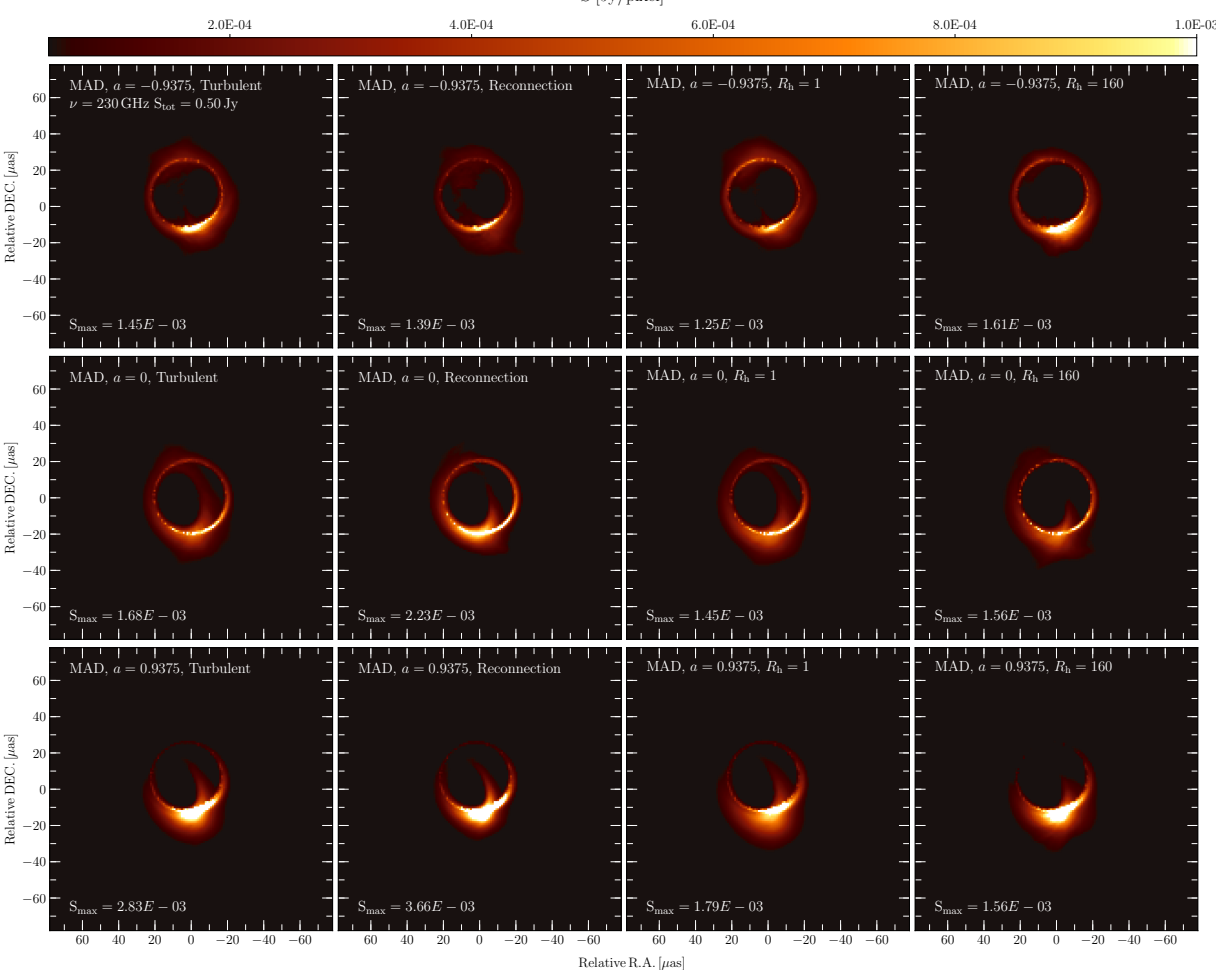

Figure 7. Same as Fig. 5, but $i=60^{\circ}$.

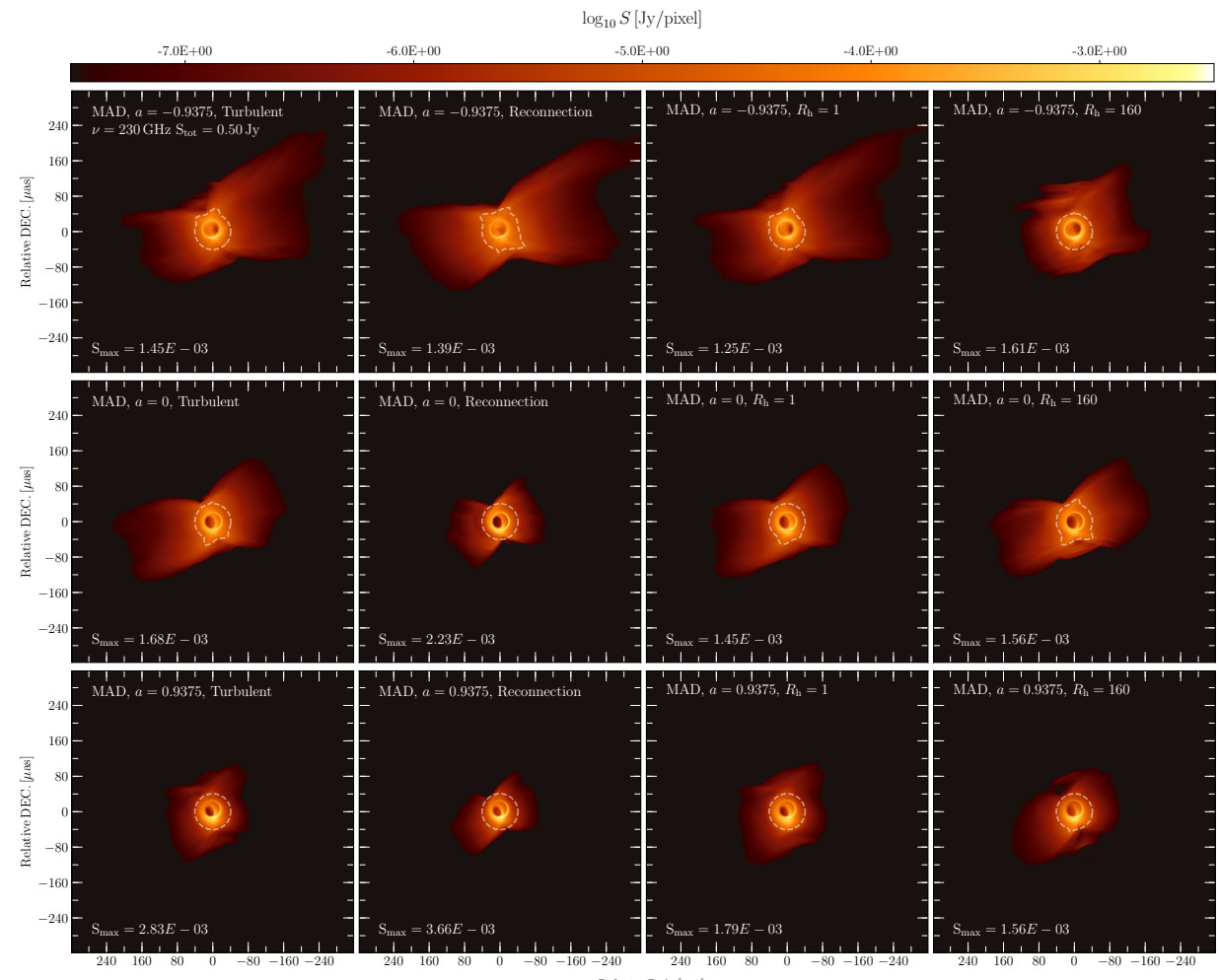

Figure 8. Same as Fig. 6, but $i=60^{\circ}$. 

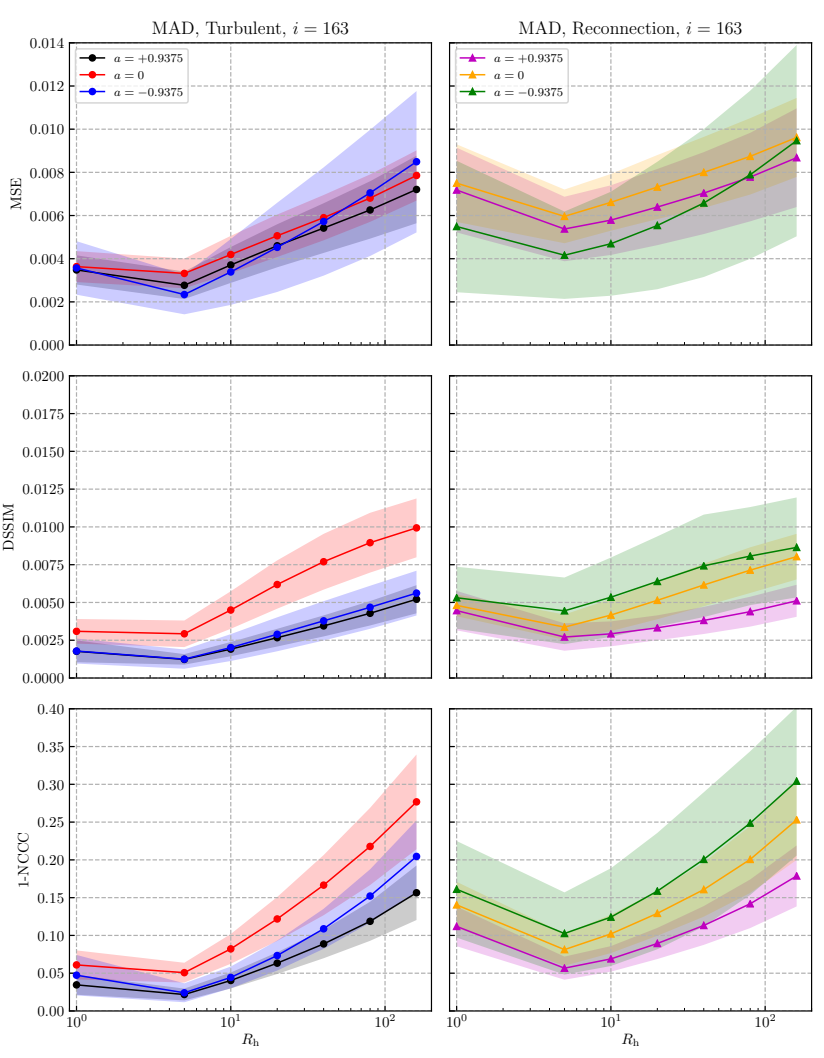

Figure 9. Image comparison distributions with different ion-to-electron ratio prescriptions using MSE (top), DSSIM (middle), and 1-NCCC (bottom). Left panels: comparison between turbulent heating and $\mathrm{R}-\beta$ prescriptions with different black-hole spins, $a=0.9375$ (black), 0 (red), and -0.9375 (blue). Right panels: comparison between magnetic-reconnection heating and R- $\beta$ prescriptions with different black-hole spins, $a=0.9375$ (magenta), 0 (orange), and 0.9375 (green). Solid lines indicate the average value and same colour bands are the standard deviation of variation within each model. In all image-comparison metrics, a smaller value corresponds to a greater similarity between compared images.

where $I_{j}$ and $K_{j}$ are the $j$-th pixels of the images $I$ and $K$ with $N$ pixels. The DSSIM is computed in terms of the human visualperception metric, also called the structural similarity index (SSIM), so that DSSIM $=1 /|\operatorname{SSIM}|-1$. Given a pair of images referred to as $I$ and $K$, the SSIM can be calculated as

$\operatorname{SSIM}(\mathrm{I}, \mathrm{K}):=\left(\frac{2 \mu_{I} \mu_{K}}{\mu_{I}^{2}+\mu_{K}^{2}}\right)\left(\frac{2 \sigma_{I K}}{\sigma_{I}^{2}+\sigma_{K}^{2}}\right)$,

where $\mu_{I}:=\sum_{j=1}^{N} I_{j} / N, \sigma_{I}^{2}=\sum_{j=1}^{N}\left(I_{j}-\mu_{j}\right)^{2} /(N-1)$, and $\sigma_{I K}:=\sum_{j=1}^{N}\left(I_{j}-\mu_{I}\right)\left(K_{j}-\mu_{K}\right) /(N-1)$. For two images, $I$ and $K$, the NCCC is computed as

$\mathrm{NCCC}:=\frac{1}{N} \sum_{j} \frac{\left(I_{j}-\langle I\rangle\right)\left(K_{j}-\langle K\rangle\right)}{\Delta_{I} \Delta_{K}}$,

where $\langle I\rangle$ and $\langle K\rangle$ are the mean pixel values in the images, and $\Delta_{I}$ and $\Delta_{K}$ are the standard deviations of the pixel values in the two images (e.g., Event Horizon Telescope Collaboration et al. 2019d). In other words, the NCCC quantifies the similarity between two images, so that NCCC $=1$ corresponds to a perfect correlation between the two images.
In these image comparisons, we use 101 snapshot GRRT images from $t=14000 M$ to $t=15000 M$ (a $10 M$ cadence) using both the aforementioned electron-heating prescriptions and the $\mathrm{R}-\beta$ model at identical simulation times. We then check the variation of each image-comparison metric. In all image-comparison metrics, smaller values mean better matching in compared images. These image comparison results for the $230 \mathrm{GHz}$ GRRT images at $i=163^{\circ}$ are presented in Fig. 9. In general, as seen in Fig. 5, the R- $\beta$ prescription in the range of $R_{\mathrm{h}}=1$ to 160 yields a good match for both electron-heating prescriptions, as is reflected in the small values for all three comparison metrics. As reference cases, we have performed two additional comparisons. The first one is between a randomly chosen GRRT image which is compared with each of the models considered. The second case makes a comparison between the time-averaged GRRT images and the individual snapshot GRRT images. Both results are summarised in Appendix B.

From the distribution of the image-comparison metrics, we do not see a clear dependence of different black-hole spin cases in either comparison with different electron-heating prescriptions, although specific average values and the variance between models are different. For the turbulent-heating prescription, the cases with $R_{\mathrm{h}}=1$ and $R_{\mathrm{h}}=5$ have the smallest values of MSE, DSSIM and 1-NCCC. Increasing the $R_{\mathrm{h}}$ value gives rise to an increase in the image-comparison metrics. For the magnetic-reconnection heating prescription, the $R_{\mathrm{h}}=5$ case has the smallest value of MSE, DSSIM and 1-NCCC. From $R_{\mathrm{h}}=5$, both metrics become larger with increasing $R_{\mathrm{h}}$ value. The smallest value of MSE ( $\left.\sim 0.004\right)$ and 1-NCCC $(\sim 0.05)$ is more than a factor 2 smaller than the vales of the comparison with a randomly chosen GRRT image (see Fig. B1). The smallest value of DSSIM is also a factor of 2 smaller than that in the comparison with a randomly chosen GRRT image in both electron-heating prescription (see Fig. B1). Note that in the majority of cases, the comparison between different ion-toelectron ratio prescriptions yields image metrics that are below those obtained from the comparison with a randomly chosen or an average image.

However, variations for each case are large. It is therefore difficult to determine which cases are best matched with a given electron-heating prescription on the basis of image-domain comparisons alone. We note that we have also applied the same imagedomain comparison for the $i=60^{\circ}$ case, obtaining similar results.

The EHT observations of a black-hole shadow image are effectively limited by the finite angular resolution. To reproduced such a limitation, we have convolved the GRRT images with a $20 \mu$ as Gaussian beam and proceeded carrying out the same comparison discussed above. Obviously, the convolved GRRT images smear out the details of the images and, as a result, the difference between the images becomes intrinsically smaller. Hence, the values of all image-comparison metrics are reduced and less dependent on value chose for $R_{\mathrm{h}}$, although they still maintain the general trend seen in Fig. 9.

Additionally, we compare the different ion-to-electron ratio prescriptions in the visibility domain. As for the image domain comparison, we again use 101 snapshot GRRT images from $t=14000 M$ to $t=15000 M$. For every image, we computed the Fourier transform, where we limited the baseline length to $10 \mathrm{G} \lambda^{4}$. From the Fourier transform, we compute the 2D distribution of the visibility amplitude (VA) and of the visibility phase (VP). Next, we calculate the mean VA and VP using all of our 101 Fourier-

\footnotetext{
4 roughly the scale the longest EHT baselines probe
} 


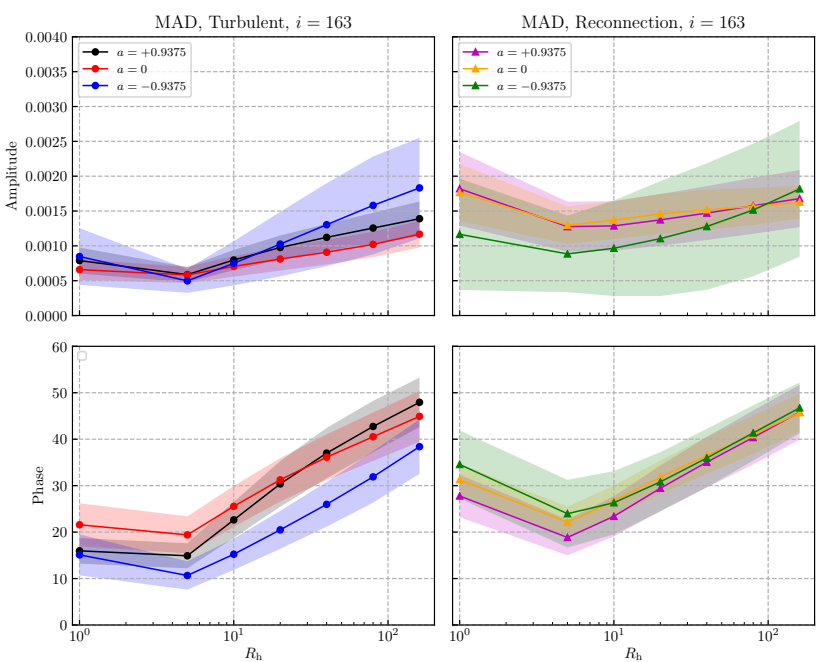

Figure 10. Distribution of comparison of different ion-to-electron ratio prescriptions in visibility amplitude (top) and phase (bottom). Left panels: comparison between turbulent heating and R- $\beta$ prescriptions with different black-hole spins, $a=0.9375$ (black), 0 (red), and -0.9375 (blue). Right panels: comparison between magnetic-reconnection heating and R- $\beta$ prescriptions with different black-hole spins, $a=0.9375$ (magenta), 0 (orange), and -0.9375 (green). Solid lines indicate average value and same colour bands indicate the standard deviation of each model.

transformed GRRT snapshots. Finally, we compute the MSE between the mean and the individual VAs and VPs. Using this procedure, we do not limit our analysis to the specific baselines (and their orientation) of current observing arrays and thus we provide an array-independent comparison of our models in Fourier space. Figure 10 shows the distribution of comparison between turbulent heating and $\mathrm{R}-\beta$ prescriptions (right panels) and between magneticreconnection heating and $\mathrm{R}-\beta$ prescriptions (left panels) in terms of visibility amplitude and phases at $i=163^{\circ}$. The general trends are the same as those seen in the image-domain comparison (see Fig. 9). A dependence on different black-hole spin cases is again not present. In the turbulent heating model, lower $R_{\mathrm{h}}$ cases have smaller differences in visibility amplitude and phase. The differences in visibility amplitude and phase become larger for larger $R_{\mathrm{h}}$ cases. In the magnetic reconnection heating prescription, the $R_{\mathrm{h}}=5$ case has a minimum in the differences of visibility amplitudes and phases.

From these quantitative comparisons of electron-heating prescriptions and $\mathrm{R}-\beta$ prescriptions in both the image and visibility domains, the $\mathrm{R}-\beta$ prescriptions are well matched to both the turbulent heating and magnetic-reconnection heating models in $230 \mathrm{GHz}$ images. In general, smaller $R_{\mathrm{h}}$ values are better matched to both heating prescriptions, but variations within each model are large. It can therefore not be said which model is better matched from the current quantitative comparisons.

\subsection{Spectral Energy Distribution}

Broadband spectral energy distributions (SEDs) generated from different ion-to-electron ratio prescriptions with different black-hole spin cases at $i=163^{\circ}$ are presented in Figure 11. In this calculation, we fix the mass and distance to correspond to M87, and the massaccretion rate is fixed so that the average total flux at $230 \mathrm{GHz}$ is $0.5 \mathrm{Jy}$. Therefore all cases have the same averaged value at $230 \mathrm{GHz}$

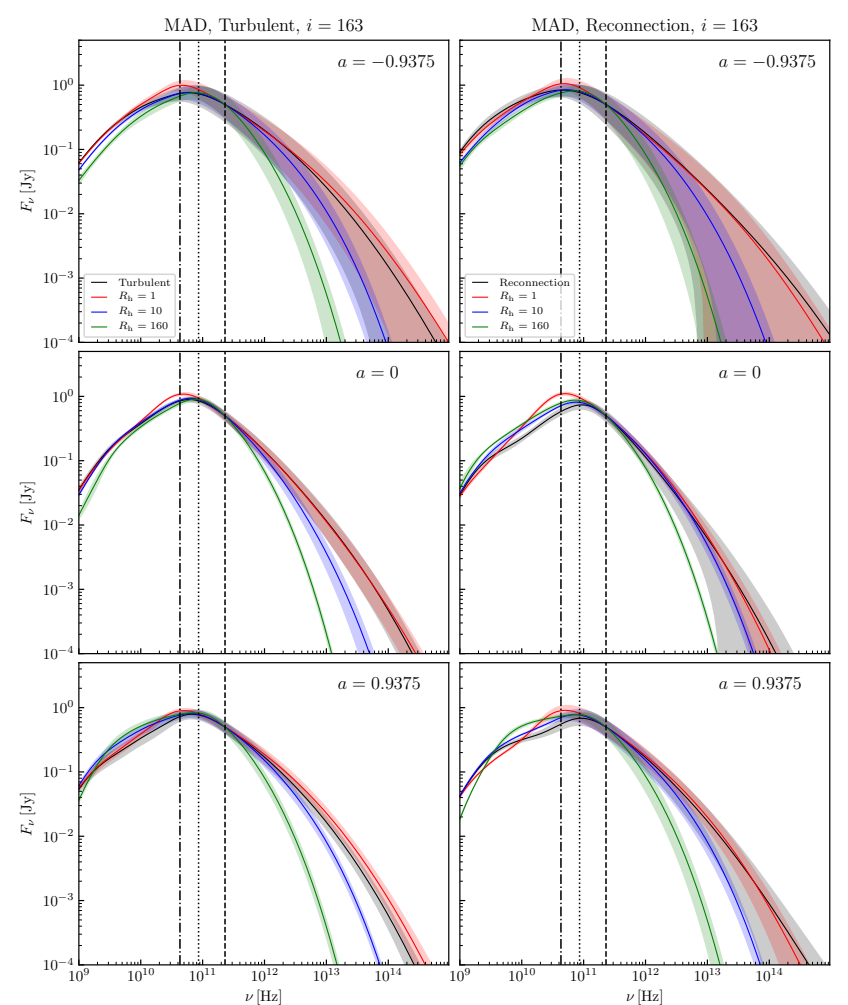

Figure 11. The spectral energy distributions (SEDs) of different ion-toelectron temperature ratio prescriptions: electron-heating model (black) and $R_{\mathrm{h}}=1$ (red), 10 (blue), and 160 (green). These are for $\mathrm{R}-\beta$ model with different black-hole spin cases (top: $a=-0.9375$, middle: 0 , and bottom: 0.9375 ) at $i=163^{\circ}$. Solid lines indicate the average value and same colour bands denote the standard deviation of time variation of the spectrum. Vertical lines indicate frequencies of 43 (dash-dotted), 86 (dotted), and $230 \mathrm{GHz}$ (dashed).

(vertical dashed line). In each model, solid lines indicates average values and shaded regions denote the standard deviation of time variation. In the $\mathrm{R}-\beta$ prescription, smaller $R_{\mathrm{h}}$ value cases have higher peak frequencies, shifted to lower frequencies. In both lower and higher frequencies, the flux typically increases with lower $R_{\mathrm{h}}$ values. This trend is generally seen in all black-hole spin cases. Time variation of SEDs is smaller in higher $R_{\mathrm{h}}$ value cases, especially at higher frequencies. For the electron-heating prescription cases, both the turbulent and the reconnection-heating prescriptions behave similarly to the $R_{\mathrm{h}}=1$ case in the $\mathrm{R}-\beta$ prescription at high frequencies, with the SED being peaked at $86 \mathrm{GHz}$. Furthermore, when comparing with the $\mathrm{R}-\beta$ model, the electron-heating prescriptions have a slightly higher flux at higher frequencies. This is reflected in the fact that electrons are hotter than ions. In the time variation of the SED, the reconnection electron-heating prescription exhibits larger time variations than turbulent electron heating at higher frequencies. From these results, we conclude that electronheating prescriptions yield similar SED profiles to lower $R_{\mathrm{h}}$ values of the $\mathrm{R}-\beta$ prescription at millimetre wavelengths. However, it will be possible to distinguish using SEDs at higher wavelengths than the sub-mm. 


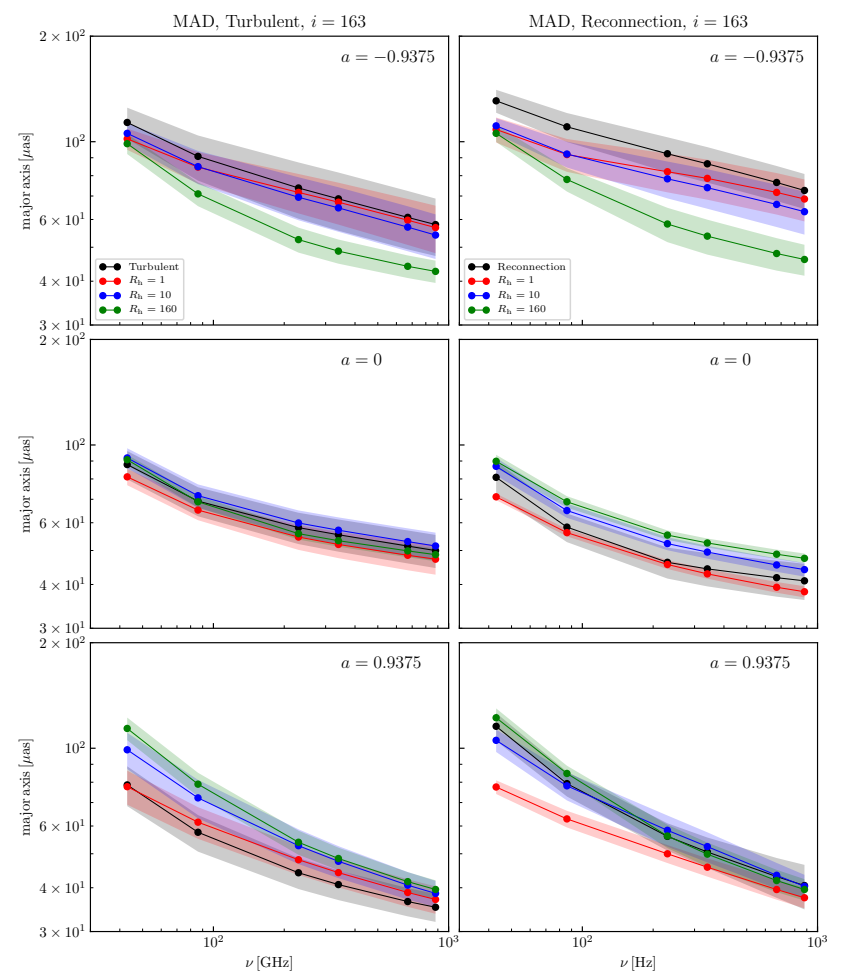

Figure 12. Image size (major axis) measured from the GRRT images at different frequencies ( $43 \mathrm{GHz}$ to $880 \mathrm{GHz}$ ) using image moments in different ion-to-electron temperature ratio prescriptions with black-hole spin $a=$ -0.9375 (top), 0 (middle), and 0.9375 (bottom) at $i=163^{\circ}$. Different colour lines indicate different ion-to-electron ratio prescriptions: turbulent heating model (black), R- $\beta$ model with $R_{\mathrm{h}}=1$ (red), 10 (blue), and 160 (green). Solid lines indicate the average value and same colour bands denote the standard deviation of time variation of the spectrum.

\subsection{Image size}

We compute the image size of the GRRT images at different frequencies $(43 \mathrm{GHz}$ to $880 \mathrm{GHz}$ ) using image moments. The semi-major and semi-minor axes of the images are computed from the eigenvalues $\lambda_{1,2}$ of the covariance matrix formed by the second-order central-image moments. The final FWHM of an equivalent elliptical Gaussian is computed as $\Theta_{\text {major } / \text { minor }}=\sqrt{8 \ln (2) \lambda_{1,2}}$ (see, e.g., Davelaar et al. 2019). The result of this analysis can be found in Fig. 12. The different panels show the size of the major axis of the ellipse enclosing the image structure for different heating models (left: turbulent heating and right: reconnection heating) for different black-hole spins (top: $a=-0.9375$, middle: $a=0$ and bottom: 0.9375). In each panel, the black curve indicate the heating model and the other colours correspond to the R- $\beta$ model. In particular, for $a=-0.94$, the electron-heating prescriptions yield a larger image size, especially at high frequencies, due to the diffused extended emission seen in Fig. 6. This result also agrees well with the larger flux densities at higher frequencies which is shown in the SED (see Fig 11).

\subsection{Time variability}

Figure 13 shows the light curve of the total flux at $230 \mathrm{GHz}$ for different ion-to-electron ratio prescriptions with different black-hole

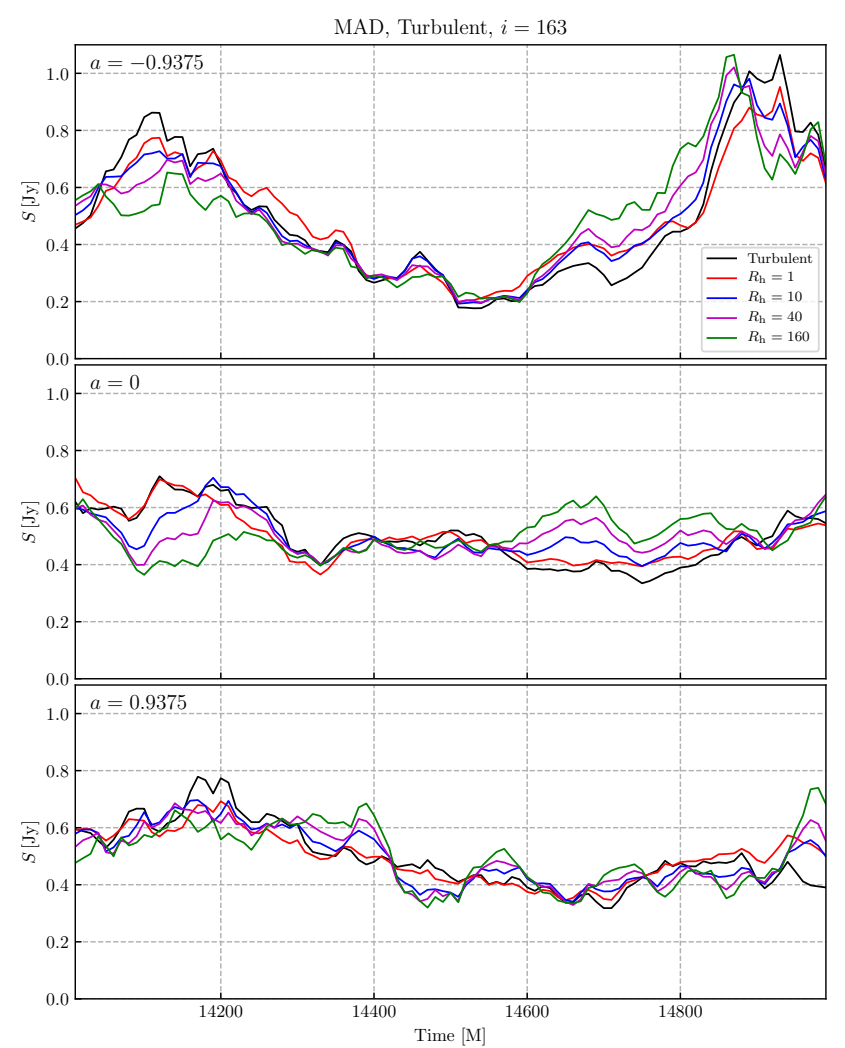

Figure 13. Light curve of total flux at $230 \mathrm{GHz}$ in different ion-to-electron temperature ratio prescriptions with black-hole spin $a=-0.9375$ (top), 0 (middle), and 0.9375 (bottom) at $i=163^{\circ}$. Different colour lines indicate different ion-to-electron ratio prescriptions: turbulent heating model (black), $\mathrm{R}-\beta$ model with $R_{\mathrm{h}}=1$ (red), 10 (blue), 40 (magenta), and 160 (green).

spin cases, at $i=163^{\circ}$. Although we see some small differences in variability at short timescales, all cases follow a trend of large variations in the light curves. The quantitative measurement of the fraction of total flux variation at $230 \mathrm{GHz}$ is shown in Fig. 14. In the comparison with different ion-to-electron temperature ratio prescriptions, dependence on the fraction of total flux variation at $230 \mathrm{GHz}$ is not seen. However, we see some dependence in different black-hole spin cases. This shows that retrograde spins have larger fractions of total flux variation at $230 \mathrm{GHz}$ than non-rotating and prograde spin cases. The non-rotating black hole case has the smallest total flux variation. This may be a consequence of the activity of orbiting flux tubes which are violent episodes of flux escape from the black hole magnetosphere (Porth et al. 2020), requiring a modestly spinning black hole. The counter-rotating black hole case typically has a larger amount of magnetic energy contained within orbiting flux tubes.

\subsection{Exclusion of Magnetised Region}

Due to the uncertainty of the results of the numerical simulations in the highly magnetised region of the polar funnels, we have necessarily excluded regions with $\sigma=1$ for the calculation of the GRRT images. Chael et al. (2019) has investigated the dependence of the different $\sigma$ thresholds on the $230 \mathrm{GHz}$ images and broadband spectra for M87 in two-temperature radiative GRMHD simulations of a MAD model. It was found that incorporating radiative feed- 

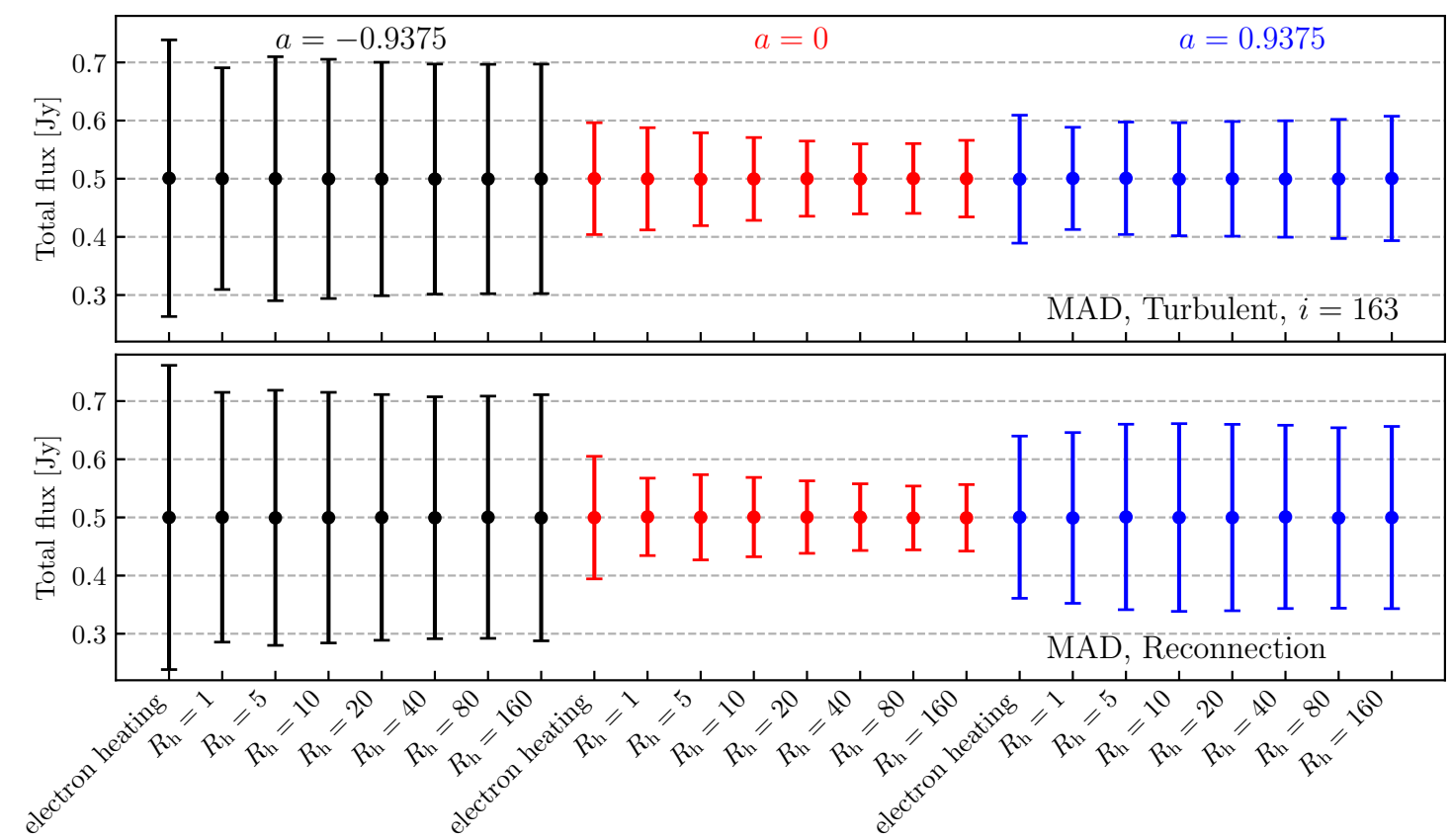

Figure 14. Fraction of total flux variation at $230 \mathrm{GHz}$ in different ion-to-electron temperature ratio prescriptions (top: turbulent heating model, bottom: reconnection heating model). Dotted point is averaged value and error bar is standard deviation of variance. Different colour indicates different black-hole spin cases, $a=-0.9375$ (red), 0 (black), and 0.9375 (blue)

back from regions with $\sigma>25$ makes the images more compact. For broadband spectra, the difference in the images produced from different choices in the $\sigma$ threshold is rather small, at least for frequencies up to $230 \mathrm{GHz}$.

Here, we investigate the impact of the exclusion of different portions of the highly magnetised regions on the image comparison. The results on the comparison for the $230 \mathrm{GHz}$ GRRT images at $i=163^{\circ}$ using different criteria for the exclusion of highly magnetised regions are presented in Fig. 15. In essence, it is clear that similar images are obtained when using different values of the cutoff $\sigma$. For both the turbulent- and the magnetic-reconnection heating prescriptions, the case with $R_{\mathrm{h}}=5$ has the smallest values of MSE, DSSIM and 1-NCCC, so that the image-comparison metrics increase with larger values of $R_{\mathrm{h}}$. Increasing the value of the cut-off $\sigma$ leads to smaller values of the image metrics, but the difference is very small. As a result, we can conclude that choosing different sigma cut-off values such as $\sigma=5$ does not significantly affect our results.

\section{DISCUSSION}

In this study, we have focused on $230 \mathrm{GHz}$ images with two different inclination angles $\left(i=60^{\circ}\right.$ and $\left.163^{\circ}\right)$. Although the emission morphology is not much different when viewed in a linear scale, the electron-heating prescriptions have a more extended and diffused emission than the R- $\beta$ prescription. Because of the limited dynamical range of the 2017 EHT observations, the images produced do not allow us to distinguish the presence of and extend jet structure, thus preventing from a clear identification of the electron-heating mechanisms at play in M87*. Such an extended emission component will be more clearly seen at lower frequencies such as $43 \mathrm{GHz}$ and 86 GHz (e.g., Chael et al. 2019; Davelaar et al. 2019; Chatter-
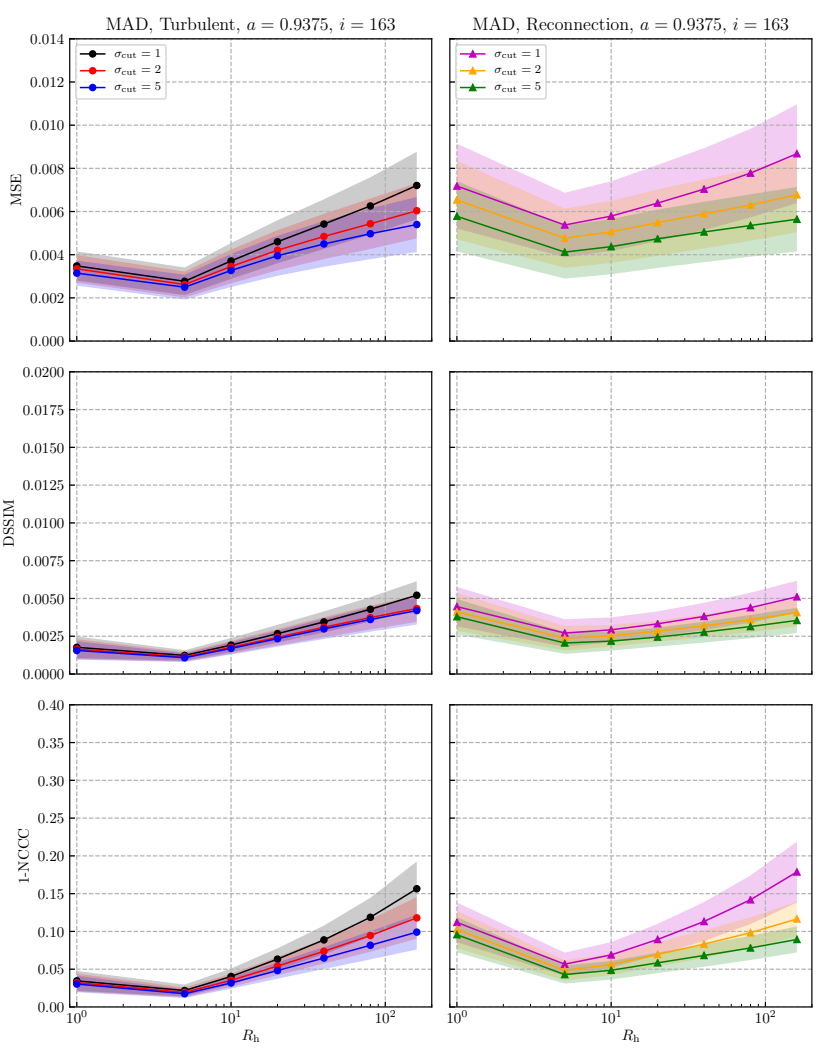

Figure 15. Same as Fig. 9 but different exclusion of magnetised region, $\sigma_{\text {cut }}=1$ (black and magenta), 2 (red and orange), and 5 (blue and green). 
jee et al. 2020). Therefore the difference between electron heating and R- $\beta$ prescriptions may be greater at longer wavelengths. Some indications of this have already been seen in the broadband SEDs for different ion-to-electron temperature ratio prescription values. Near future observations $230 \mathrm{GHz}$ - performed either by the nextgeneration EHT (ngEHT) or from space (Doeleman et al. 2019; Raymond et al. 2021; Roelofs et al. 2021) - are expected to improve the both the coverage in the $(u, v)$ plane and the dynamical range, thus allowing for the detection of a diffused extended-jet structure and, in turn, providing the evidence to distinguish the different potential electron-heating mechanism.

Using a relativistic thermal electron distribution function is one of the limitations of the present study, which will break down in regions where non-ideal effects for magnetic fields are important. These non-ideal effects are expected to be strong in highly magnetised regions such as the jet funnel, where they can be associated with electron acceleration mechanisms such as magnetic reconnection or turbulence. Davelaar et al. $(2018,2019)$ use a $\kappa$ electron distribution function, which is a combination of a thermal core with a power-law tail at larger electron Lorentz factors, for GRMHD simulations of M87. Although images at $230 \mathrm{GHz}$ do not show much difference, non-thermal emission will produce more prominent and extended emission at lower frequencies such as $43 \mathrm{GHz}$ and $86 \mathrm{GHz}$ or at a higher frequencies as $1.1 \mathrm{THz}$ (Petersen \& Gammie 2020). Distributions like the $\kappa$ electron distribution function yield a better fit of the broadband spectrum of M87 (Davelaar et al. 2019), which we will investigate in the context of the present electron-heating prescription for thermal core in a future work.

Our choice of accretion model, the MAD model, is the extreme limit of high magnetic flux accretion onto a black hole horizon. Another typical model is so-called Standard Accretion and Normal Evolution (SANE; Narayan et al. (2012); Sądowski et al. (2013)). As seen in Event Horizon Telescope Collaboration et al. (2019e), GRRT images of SANE models at $230 \mathrm{GHz}$ have a greater dependence on $R_{\mathrm{h}}$ values than MAD models. Differences between electron-heating prescriptions and $\mathrm{R}-\beta$ models may be larger than the current comparisons in MAD models and these considerations prevent us from making here more general statements that may be falsified under different accretion conditions. We will perform a similar investigation of the comparison of different ion-to-electron temperature ratio prescriptions to extended SANE models in an upcoming paper.

In the theoretical comparison of the observations of M87* by the Event Horizon Telescope Collaboration et al. (2019e), the large majority of the GRMHD models produced were considered compatible with the observed shadow image. At the same time, a class of these models were rejected when three additional constraints were imposed, namely: a consistent radiative efficiency, no overproduction of X-ray emission, and jet power compatible with large-scale radio observations. More specifically, the radiative efficiency was calculated as $\epsilon \equiv L_{\mathrm{bol}} / \dot{M} c^{2}$, where $L_{\mathrm{bol}}$ is the bolometric luminosity. If such an efficiency was found to be larger than that of a thin, radiatively efficient disc (Novikov \& Thorne 1973), i.e., $\epsilon>0.2$, then the model was rejected. In this way, a number of MAD-accretion models with low values of $R_{\mathrm{h}}$ were ruled-out because they were found to be radiatively inconsistent. This result, however, was mostly due to the very low mass-accretion rates of these models, which had $\dot{M} / \dot{M}_{\text {Edd }} \leq 10^{-6}$ and thus resulted in being radiatively inefficient. However, the mass-accretion rate in our MAD-model simulations with a low $R_{\mathrm{h}}$ is around $10^{-5} \dot{M}_{\text {Edd }}$ (see Appendix D), i.e., one order of magnitude greater than that found by the Event Horizon Telescope Collaboration et al. (2019e). As a result, the corresponding radiative efficiency of all of our MAD models - even those with a low $R_{\mathrm{h}}$ - is still compatible with the constraints from a thin disc, and hence they can all be considered compatible with the observations. There are a number of potential sources for the different mass-accretion rates measured both here and those considered by Event Horizon Telescope Collaboration et al. (2019e), but the different adiabatic index $\left(\Gamma_{\mathrm{g}}=4 / 3\right.$ versus $\Gamma_{\mathrm{g}}=13 / 9$ in the previous work) is likely the element most responsible for this difference.

We also note that a value of $\Gamma_{\mathrm{g}}=4 / 3$ reflects the assumption that the plasma is relativistic. The initial gas torus might be cooler and have a higher adiabatic index, e.g., $\Gamma_{g} \simeq 5 / 3$. As seen in Appendix $\mathrm{A}$, differences in adiabatic index between gas and electrons affects the efficiency of the electron heating. This effect is seen in the dimensionless electron temperature distribution seen in Fig. 2 and in previous studies (Chael et al. 2019; Dexter et al. 2020) However, the GRRT images we calculate have the same general trend as those seen in Chael et al. (2019). We therefore conclude that our results are not affected appreciably by our choice of adiabatic index, but they may be affected by the measurement of the mass-accretion rate.

\section{CONCLUSIONS}

In this paper, we have investigated the commonly used ion-toelectron temperature ratio prescription, the R- $\beta$ model, by computing GRRT images at $230 \mathrm{GHz}$ which serve to facilitate comparison between electron-heating prescriptions obtained from GRMHD simulations with electron thermodynamics. From the comparison of GRRT images, the R- $\beta$ prescription in the range of $R_{\mathrm{h}}$ from 1 to 160 with fixed $R_{1}=1$ is well-matched by both turbulent heating and magnetic-reconnection heating prescriptions, although images of electron-heating prescriptions have a more extended and diffused emission region. From this comparison study of different physical aspects, including images, visibilities, broadband spectra, and light curves, we conclude that the commonly-used R- $\beta$ model favourably reproduces the ion-to-electron temperature prescription obtained from electron thermodynamics calculations of accretion flows onto a black hole at $230 \mathrm{GHz}$. In general, smaller $R_{\mathrm{h}}$ values yield a better match to both heating prescriptions. For observations at longer wavelengths, such as $43 \mathrm{GHz}$ or $86 \mathrm{GHz}$, a greater difference in the images due to the more extended and diffused emission in the electron-heating prescription cases is expected, in particular for the counter-rotating accretion. We note that our conclusions apply to MAD accretion models, and it is expected that greater differences will be found for SANE models, which exhibit much greater variability than MAD models.

\section{ACKNOWLEDGEMENTS}

We would like to thank Alejandro Cruz Osorio, Antonios Nathanail, Jonas Köhler, Roman Gold, Mariafelicia de Laurentis, and Avery Broderick, for useful discussions. This research is supported by the ERC synergy grant "BlackHoleCam: Imaging the Event Horizon of Black Holes" (grant number 610058). CMF is supported by the Black Hole Initiative at Harvard University, which is supported by a grant from the John Templeton Foundation. ZY is supported by a Leverhulme Trust Early Career Fellowship. The simulations were performed on GOETHE at the CSC-Frankfurt, Iboga at ITP 
Frankfurt, and Pi2.0 at Shanghai Jiao Tong University. This research has made use of NASA's astrophysics data system (ADS).

\section{DATA AVAILABILITY}

The data underlying this article will be shared on reasonable request to the corresponding author.

\section{REFERENCES}

Anantua R., Ressler S., Quataert E., 2020, Mon. Not. R. Astron. Soc., 493, 1404

Bower G. C., Wright M. C. H., Falcke H., Backer D. C., 2003, Astrophys. J., 588, 331

Broderick A. E., Loeb A., 2006, Astrophys. J. Lett.,, 636, L109

Broderick A. E., Fish V. L., Doeleman S. S., Loeb A., 2009, Astrophys. J., 697,45

Broderick A. E., Fish V. L., Doeleman S. S., Loeb A., 2011, Astrophys. J., 735,110

Broderick A. E., et al., 2016, Astrophys. J., 820, 137

Chael A., Rowan M., Narayan R., Johnson M., Sironi L., 2018, Mon. Not. R. Astron. Soc., 478, 5209

Chael A., Narayan R., Johnson M. D., 2019, Monthly Notices of the Royal Astronomical Society, 486, 2873

Chan C.-k., Psaltis D., Özel F., Medeiros L., Marrone D., Saḑowski A., Narayan R., 2015, Astrophys. J., 812, 103

Chatterjee K., et al., 2020, arXiv e-prints, p. arXiv:2002.08386

Davelaar J., Mościbrodzka M., Bronzwaer T., Falcke H., 2018, Astron. Astrophys., 612, A34

Davelaar J., et al., 2019, Astron. Astrophys., 632, A2

Dexter J., Agol E., Fragile P. C., 2009, Astrophys. J.1, 703, L142

Dexter J., Agol E., Fragile P. C., McKinney J. C., 2010, Astrophys. J., 717, 1092

Dexter J., et al., 2020, Mon. Not. R. Astron. Soc., 494, 4168

Doeleman S. S., et al., 2008, Nature, 455, 78

Doeleman S., et al., 2019, in Bulletin of the American Astronomical Society. p. $256(\operatorname{arXiv:1909.01411)}$

Event Horizon Telescope Collaboration et al., 2019a, Astrophys. J. Lett., 875, L1

Event Horizon Telescope Collaboration et al., 2019b, Astrophys. J. Lett., 875, L2

Event Horizon Telescope Collaboration et al., 2019c, Astrophys. J. Lett., 875, L3

Event Horizon Telescope Collaboration et al., 2019d, Astrophys. J. Lett., $875, \mathrm{~L} 4$

Event Horizon Telescope Collaboration et al., 2019e, Astrophys. J. Lett., 875, L5

Event Horizon Telescope Collaboration et al., 2019f, Astrophys. J. Lett., 875, L6

Fishbone L. G., Moncrief V., 1976, Astrophys. J., 207, 962

Fromm C. M., Mizuno Y., Younsi Z., Olivares H., Porth O., De Laurentis M., Rezzolla L., 2020, Astron. Astrophys., submitted

Goddi C., et al., 2017, International Journal of Modern Physics D, 26, 1730001

Gold R., McKinney J. C., Johnson M. D., Doeleman S. S., 2017, Astrophys. J., 837,180

Hada K., et al., 2017, Publications of the ASJ, 69, 71

Ho L. C., 2009, Astrophys. J., 699, 626

Howes G. G., 2010, Mon. Not. R. Astron. Soc., 409, L104

Kawazura Y., Barnes M., Schekochihin A. A., 2019, Proceedings of the National Academy of Science, 116, 771

Kawazura Y., Schekochihin A. A., Barnes M., TenBarge J. M., Tong Y., Klein K. G., Dorland W., 2020, arXiv e-prints, p. arXiv:2004.04922

Kim J., et al., 2018, The Astrophysical Journal, 861, 129

Kuo C. Y., et al., 2014, Astrophys. J. Lett., 783, L33
Leung P. K., Gammie C. F., Noble S. C., 2011, Astrophys. J., 737, 21

Mahadevan R., 1998, Nature, 394, 651

Mahadevan R., Quataert E., 1997, Astrophys. J., 490, 605

Marrone D. P., Moran J. M., Zhao J.-H., Rao R., 2007, Astrophys. J.1, 654, L57

McKinney J. C., Tchekhovskoy A., Blandford R. D., 2012, Mon. Not. R. Astron. Soc., 423, 3083

Mizuno Y., et al., 2018, Nature Astronomy, 2, 585

Mościbrodzka M., Gammie C. F., Dolence J. C., Shiokawa H., Leung P. K., 2009, Astrophys. J., 706, 497

Mościbrodzka M., Shiokawa H., Gammie C. F., Dolence J. C., 2012, Astrophys. J. Lett., 752, L1

Mościbrodzka M., Falcke H., Shiokawa H., Gammie C. F., 2014, Astron. Astrophys., 570, A7

Mościbrodzka M., Falcke H., Shiokawa H., 2016, Astron. Astrophys., 586, A38

Narayan R., Yi I., 1994, Astrophys. J. Lett., 428, L13

Narayan R., Igumenshchev I. V., Abramowicz M. A., 2003, Publications of the ASJ, 55, L69

Narayan R., Sądowski A., Penna R. F., Kulkarni A. K., 2012, Mon. Not. R. Astron. Soc., 426, 3241

Noble S. C., Leung P. K., Gammie C. F., Book L. G., 2007, Classical and Quantum Gravity, 24, S259

Novikov I. D., Thorne K. S., 1973, in Black Holes (Les Astres Occlus). pp $343-450$

Olivares H., Porth O., Davelaar J., Most E. R., Fromm C. M., Mizuno Y., Younsi Z., Rezzolla L., 2019, Astron. Astrophys., 629, A61

Petersen E., Gammie C., 2020, Mon. Not. R. Astron. Soc., 494, 5923

Porth O., Olivares H., Mizuno Y., Younsi Z., Rezzolla L., Moscibrodzka M., Falcke H., Kramer M., 2017, Computational Astrophysics and Cosmology, 4, 1

Porth O., et al., 2019, Astrophys. J. Supp., 243, 26

Porth O., Mizuno Y., Younsi Z., Fromm C. M., 2020, Mon. Not. R. Astron. Soc., in prep.

Prieto M. A., Fernández-Ontiveros J. A., Markoff S., Espada D., GonzálezMartín O., 2016, Mon. Not. R. Astron. Soc., 457, 3801

Pu H.-Y., Broderick A. E., 2018, Astrophys. J., 863, 148

Pu H.-Y., Yun K., Younsi Z., Yoon S.-J., 2016, Astrophys. J., 820, 105

Raymond A. W., et al., 2021, Astrophys. J., Supp., 253, 5

Ressler S. M., Tchekhovskoy A., Quataert E., Chand ra M., Gammie C. F., 2015, Mon. Not. R. Astron. Soc., 454, 1848

Ressler S. M., Tchekhovskoy A., Quataert E., Gammie C. F., 2017, Mon. Not. R. Astron. Soc., 467, 3604

Rezzolla L., Zanotti O., 2013, Relativistic Hydrodynamics. Oxford University Press, Oxford, UK, doi:10.1093/acprof:oso/9780198528906.001.0001

Roelofs F., Fromm C. M., Mizuno Y., Davelaar J., Janssen M., Younsi Z., Rezzolla L., Falcke H., 2021, arXiv e-prints, p. arXiv:2103.16736

Rowan M. E., Sironi L., Narayan R., 2017, Astrophys. J., 850, 29

Rowan M. E., Sironi L., Narayan R., 2019, Astrophys. J., 873, 2

Ryan B. R., Ressler S. M., Dolence J. C., Tchekhovskoy A., Gammie C., Quataert E., 2017, Astrophys. J. Lett., 844, L24

Ryan B. R., Ressler S. M., Dolence J. C., Gammie C., Quataert E., 2018, Astrophys. J., 864, 126

Shcherbakov R. V., Penna R. F., McKinney J. C., 2012, Astrophys. J., 755, 133

Sądowski A., Narayan R., Penna R., Zhu Y., 2013, Mon. Not. R. Astron. Soc., 436, 3856

Sądowski A., Wielgus M., Narayan R., Abarca D., McKinney J. C., Chael A., 2017, Mon. Not. R. Astron. Soc., 466, 705

Tchekhovskoy A., McKinney J. C., 2012, Mon. Not. R. Astron. Soc., 423, L55

Tchekhovskoy A., Narayan R., McKinney J. C., 2011, Mon. Not. R. Astron. Soc., 418, L79

Walker R. C., Hardee P. E., Davies F. B., Ly C., Junor W., 2018, Astrophys. J., 855, 128

Wang Z., Bovik A. C., Sheikh H. R., Simoncelli E. P., 2004, IEEE Transactions on Image Processing, 13, 600 
Younsi Z., Wu K., Fuerst S. V., 2012, Astron. Astrophys., 545, A13

Younsi Z., Porth O., Mizuno Y., Fromm C. M., Olivares H., 2020. pp 9-12, doi: $10.1017 / \mathrm{S} 1743921318007263$

Yuan F., Narayan R., 2014, Annual Review of Astronomy and Astrophysics, 52,529

\section{APPENDIX A: 1D NOH SHOCK TEST}

We demonstrate the validity and convergence properties of our implementation of the electron-heating prescription using a 1D Noh shock test problem. The Noh shock test examines the problem of shock reflection. The time-dependent solution consists of two shocks originating at the initial discontinuity and travelling to the left and right boundaries.

In high Mach number shocks, the electrons receive a constant fraction of the viscous heating by the shock. As seen in Ressler et al. (2015), the post-shock electron internal energy $u_{e}^{f}$ is given by

$\frac{u_{\mathrm{e}}^{f}}{u_{\mathrm{g}}^{f}}=\frac{f_{\mathrm{e}}}{2}\left[\left(\frac{\Gamma_{\mathrm{g}}+1}{\Gamma_{\mathrm{g}}-1}\right)^{\Gamma_{\mathrm{e}}}\left(1-\frac{\Gamma_{\mathrm{g}}}{\Gamma_{\mathrm{e}}}\right)+1+\frac{\Gamma_{\mathrm{g}}}{\Gamma_{\mathrm{e}}}\right] \frac{\Gamma_{\mathrm{g}}^{2}-1}{\Gamma_{\mathrm{e}}^{2}-1}$,

where $u_{\mathrm{g}}^{f}$ is post-shock internal energy of the fluid. When $\Gamma_{\mathrm{g}}=\Gamma_{\mathrm{e}}$, $u_{\mathrm{e}}^{f} / u_{\mathrm{g}}^{f}$ is equal to $f_{\mathrm{e}}$. When we choose $\Gamma_{\mathrm{g}}=5 / 3$ and $\Gamma_{\mathrm{e}}=4 / 3$, $u_{\mathrm{e}}^{f} / u_{\mathrm{g}}^{f}$ becomes $\sim 0.76 f_{\mathrm{e}}$.

In our simulations, we assume an unmagnetised, nonrelativistic $\left(\Gamma_{\mathrm{g}}=5 / 3\right)$ cold fluid with uniform density and gas pressure as an initial condition. The initial velocities have discontinuities at the half of computational domain (left and right states) and are directed towards the discontinuous boundary with non-relativistic speed $|v|=0.001 \mathrm{c}$. This creates a strong shock $(M \gg 1)$ propagating in the left and right directions. In this test, $f_{\mathrm{e}}$ is fixed as 0.5 and the test is performed for both $\Gamma_{\mathrm{e}}=5 / 3$ and $\Gamma_{\mathrm{e}}=4 / 3$.

Figure A 1 shows the distribution of density and the ratio of internal energy between electrons and gas at $t=700$. Two shocks propagate at $x \approx 0.25$ and 0.75 . After the shock, the density jumps to a value four times larger than the pre-shock region, which indicates a strong shock has been created. In the $\Gamma_{\mathrm{e}}=5 / 3$ case, the ratio of internal energy between electrons and gas at the post-shock region is in good agreement with the analytical solution. However, for the $\Gamma_{\mathrm{e}}=4 / 3$ case there is not as close a match with the analytical solution and differs by $\sim 4 \%$. Similar results are also seen in Ressler et al. (2015). This is because the correct heating by the shock needs the shock structure to be well-resolved. In our numerical scheme, the shock structure is still resolved with a few grid points. We therefore cannot resolve the shock completely, even at higher grid resolutions than those adopted in this study. For the $\Gamma_{\mathrm{g}}=\Gamma_{\mathrm{e}}$ case, the density term is cancelled out in the time evolution of the electron entropy, and its dependence on the shock structure may be neglected.

The simulation convergence for the electron heating calculations is shown in Fig. A2. As expected, the $\Gamma_{\mathrm{e}}=5 / 3$ case converges at first order (i.e., as $1 / N$, where $N$ is the number of cells used), but the $\Gamma_{\mathrm{e}}=4 / 3$ case does not converge to the analytical solution, as seen in Fig. A1 (numerical results differ by $\sim 4 \%$ ). In order to reach the analytical solution in the $\Gamma_{\mathrm{g}} \neq \Gamma_{\mathrm{e}}$ case, we would need to introduce dissipative effects and in particular a bulk viscosity Ressler et al. (2015). Given that we are concerned primarily with synthetic $230 \mathrm{GHz}$ images, $a \leq 4 \%$ error is acceptable for the present study.

\section{APPENDIX B: IMAGE COMPARISON TEST}

In order to provide a reference value of the image-comparison metrics, we consider the comparison between each prescription model ( different $R_{\mathrm{h}}$ value of $R-\beta$ parameterised prescriptions) and a randomly chosen single image. For the latter, we consider the $230 \mathrm{GHz}$ GRRT image of a black hole with spin $a=0$ using the $R-\beta$ parameterised prescription with $R_{\mathrm{h}}=40$ at $t=15000 M$.

Figure B1 shows the results of the image comparison for the $230 \mathrm{GHz}$ GRRT images at $i=163^{\circ}$ with a black-hole spin $a=0.9375$. From the distribution of the image-comparison metrics, no evidence emerges for a clear dependence on the different heating prescriptions. In other words, all models match the chosen reference image equally poorly or favourably. The average values of the metrics MSE, DSSIM, and 1-NCCC are 0.08-0.09, 0.01, and $0.25-0.3$, respectively. Note that the MSE has a slightly larger variance for larger values of $R_{\mathrm{h}}$. More importantly, these average values are around a factor of two larger than the smallest value in Fig. 9. We also note that the correlation time of images in GRMHD simulations has been found to be around $50 \mathrm{M}$, that is, any two random images of a GRMHD simulation do not show a correlation if the corresponding time separation is larger than $50 \mathrm{M}$. Beyond this window in time, the differences can be very significant.

Another reference value of the image-comparison metrics is obtained by the comparison between time-averaged GRRT images and individual snapshot GRRT images in each prescription model with different black hole spin. This is shown in Fig. B2. Note that all distributions are essentially flat, indicating a very weak dependence on the value of $R_{\mathrm{h}}$. When $R_{\mathrm{h}}$ increases, the values of the MSE and 1-NCCC metrics also increase, although only slightly. Note also that the distribution of the MSE metric has a large variance in the case of counter-rotating black holes, indicating that each individual image can have a large variation from the averaged one. This behaviour was already encountered in the fraction of the total-flux variation in Fig. 14.

\section{APPENDIX C: DIFFERENT $R_{\mathrm{L}}$ CASE}

We recall that the parameterised $\mathrm{R}-\beta$ prescription for the electronion temperature ratio has two parameters, $R_{\mathrm{l}}$ and $R_{\mathrm{h}}$. As mentioned in the main text, we have kept $R_{1}=1$ fixed and varied $R_{\mathrm{h}}$. In this appendix, we investigate the effect of actually using different values of $R_{1}$. In particular, Fig. C1 presents the time-averaged GRRT images at $i=163^{\circ}$ relative to MAD simulations with a black hole spin $a=0.9375$ using the $R-\beta$ prescription with $R_{\mathrm{h}}=1$ and $R_{1}=0.1,1$, and 10 , both in a linear and in a logarithmic scale. Following Figs. 5 and 6, the images are averaged from $t=14000 \mathrm{M}$ to $15000 M$, and all averaged images have the same total flux of $0.5 \mathrm{Jy}$. Note that for values of $R_{1}$ the bright photon ring becomes dimmer and a larger amount of an extended and diffused emission is seen. On other hand, for large values of $R_{1}$ the emission is more concentrated near the photon ring and the extended emission less pronounced.

The image comparison results for the $230 \mathrm{GHz}$ GRRT images at $i=163^{\circ}$ are shown in Fig. C2. Overall, when $R_{1}$ is large, all of the three image-comparison metrics become worse, in particular for the $R_{\mathrm{h}}=1$ case. This occurs since the emission structure differs significantly from the heating-prescription case. On the other hand, if $R_{1} \lesssim 1$, all of the three metrics increase. This could be understood as due to the extended emission, which is even more extended than in the electron-heating prescriptions. This behaviour does not change 

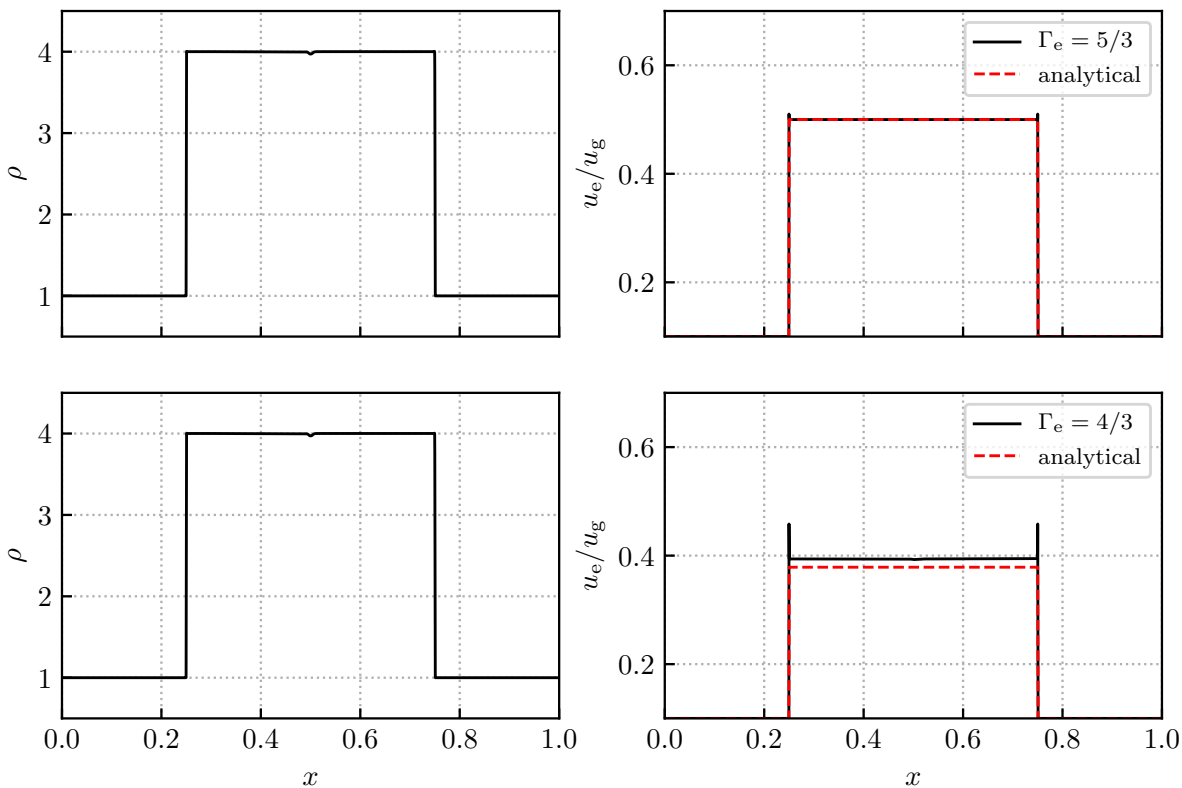

Figure A1. 1D Noh shock results for an electron heating fraction $f_{\mathrm{e}}=0.5$ using 2000 cells with $($ top $) \Gamma_{\mathrm{e}}=5 / 3$ and $($ bottom $) \Gamma_{\mathrm{e}}=4 / 3$. Left panels show density, right panels present $u_{\mathrm{e}} / u_{\mathrm{g}}$. Red dashed lines indicate analytical solutions.

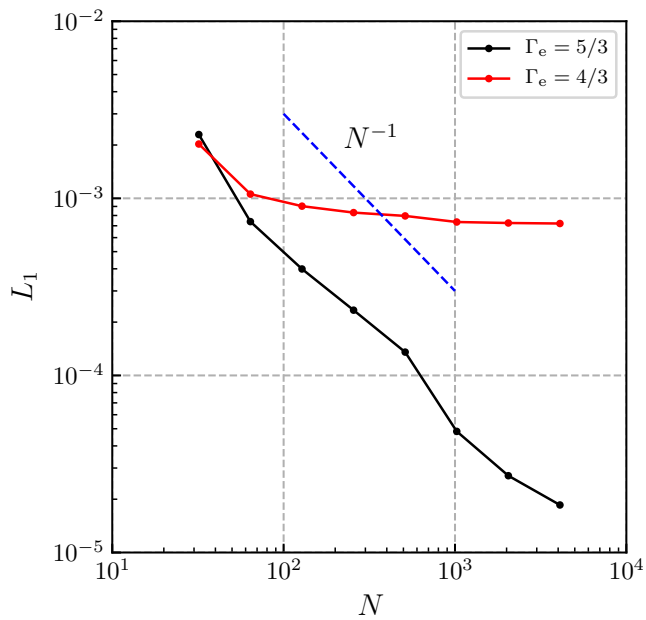

Figure A2. L1 norm of the error in the ratio of the electron-to-gas internal energies between the numerical and the analytical solutions shown as a function of the number $N$ of cells used.

considerably when considering different values of $R_{\mathrm{h}}$. Hence, from these results we conclude that our default value $R_{1}=1$ effectively provides a very good match with the electron-heating prescriptions.

\section{APPENDIX D: MASS-ACCRETION RATE}

We recall that although the GRMHD simulations are scale-free, the GRRT calculations depend on the physical scale set by the mass of the black hole. In this work, our reference black hole is M87*. A mass-scale unit is needed in the conversion from the value of the restmass employed in the simulations to a physical rest-mass density; this is done by normalising the time-averaged flux at $230 \mathrm{GHz}$ to the value of $0.5 \mathrm{Jy}$. Once a physical value for the rest-mass density is obtained, we can calculate a physical mass-accretion rate.

Figure D1 shows the mass-accretion rates normalised by the Eddington mass-accretion rate in different models. Clearly, the mass-accretion rate increases monotonically with the values of $R_{\mathrm{h}}$ value and decreases as the black-hole spin goes from maximally counter-rotating to maximally co-rotating; this latter behaviour is possibly due to the increase of the ISCO in the counter-rotating case, which allows larger-density material to be accreted. Overall, the mass-accretion rate is in the range from $10^{-6}$ to $10^{-4}$ times Eddington accretion rate. Interestingly, higher $\sigma$ cutoffs lead to lower mass-accretion rates; this is probably because when using a larger $\sigma$ cutoff we are including larger regions with high magnetisation, where the rest-mass densities are small but contribute to reaching the same reference flux.

This paper has been typeset from a $\mathrm{T}_{\mathrm{E}} \mathrm{X} / \mathrm{L} \mathrm{A} \mathrm{T} \mathrm{X}$ file prepared by the author. 

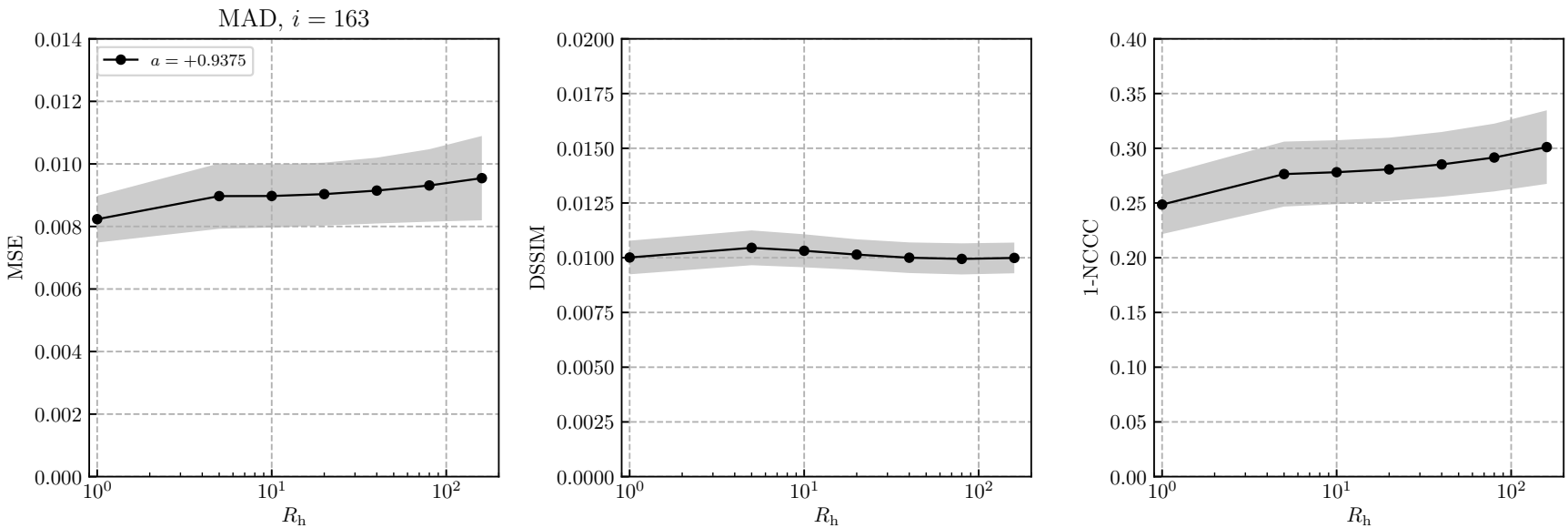

Figure B1. Image-comparison distributions at an inclination angle $i=163^{\circ}$ for different values of $R_{\mathrm{h}}$ of the $\mathrm{R}-\beta$ prescription for a black hole with spin $a=0.9375$ and a single image relative to a black hole with spin $a=0$ with $R_{\mathrm{h}}=40$ at $t=15000 M$. The different panels refer to the different metrics: MSE (right), DSSIM (middle), and 1-NCCC (left). Solid lines indicate the average values and the shaded bands are the standard deviation of the variation within each model.
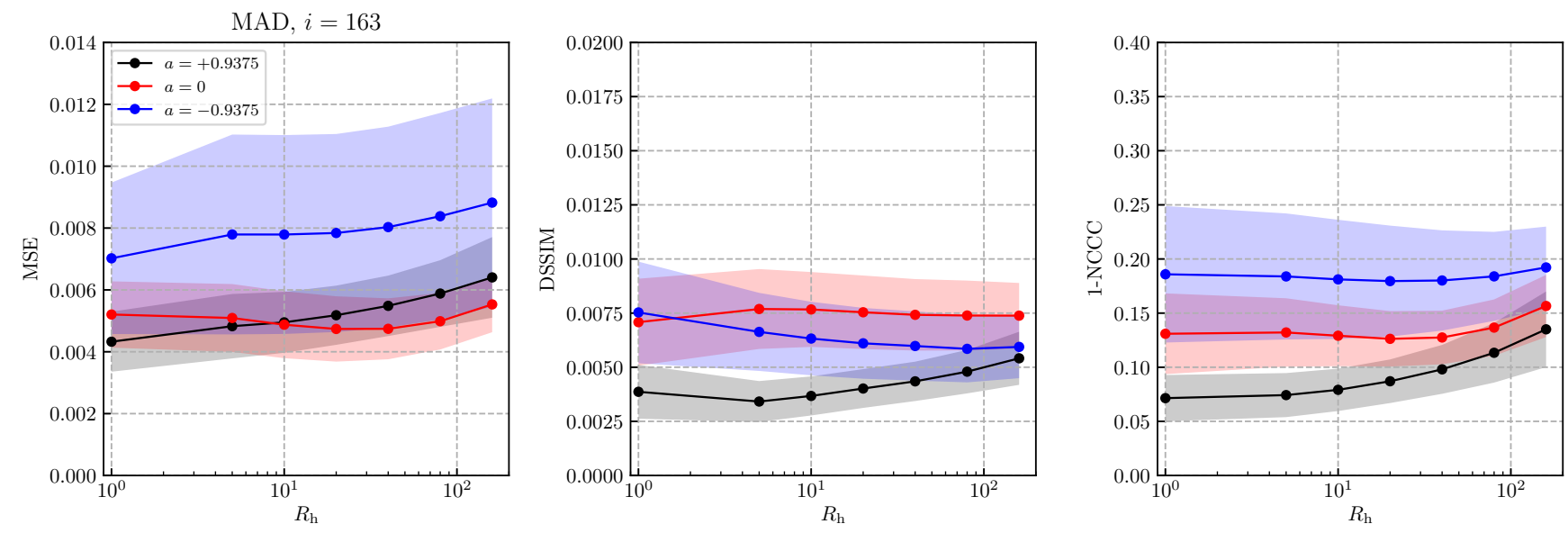

Figure B2. Same as in Fig. B1 but with the addition of the comparison with averaged images for black holes with different spins: blue for $a=-0.9375$, red for $a=0$, and black for $a=0.9375$. 

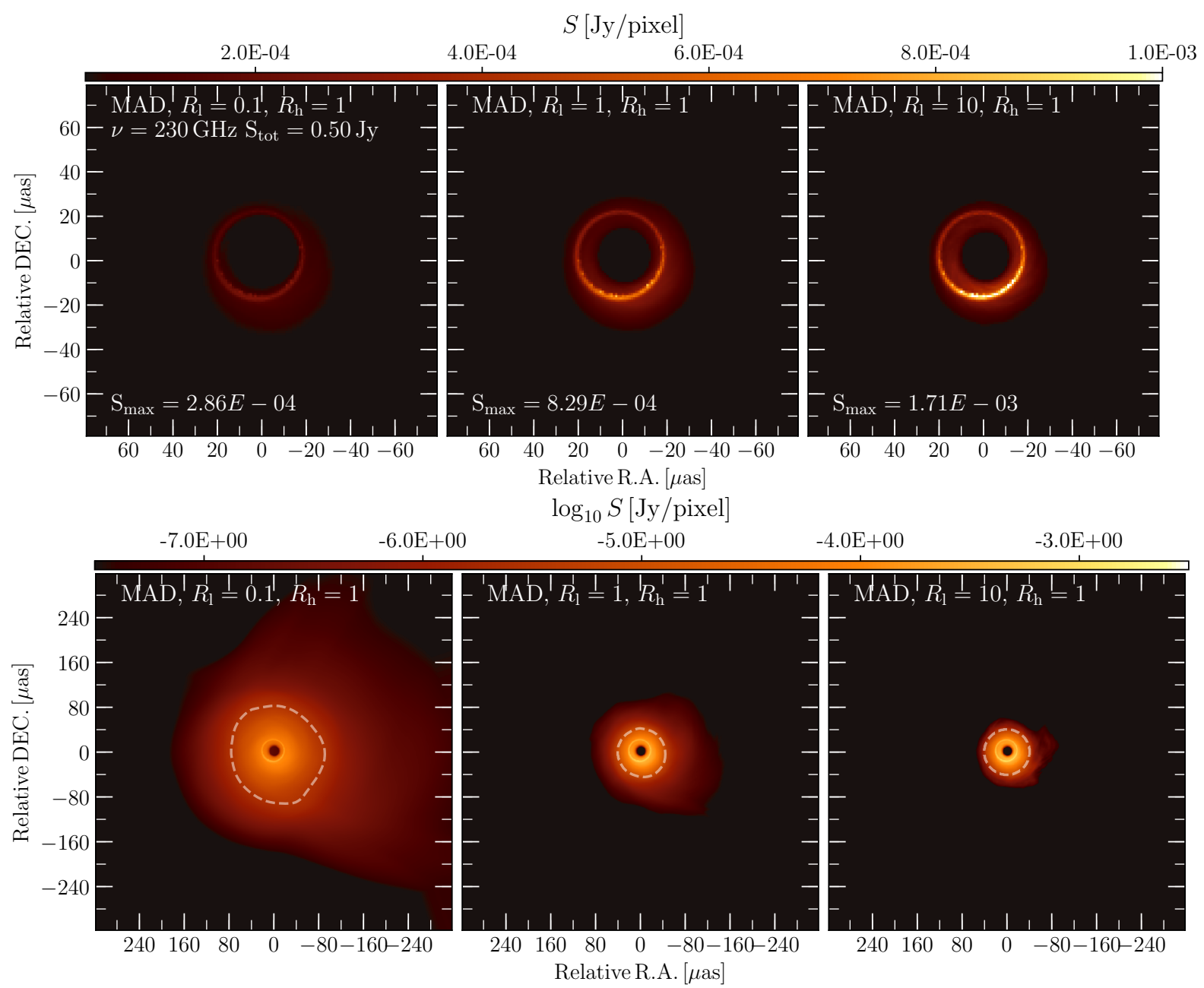

Figure C1. Time-averaged GRRT images at $i=163^{\circ}$ of MAD simulations with a black hole spin $a=0.9375$ using the $R-\beta$ prescription with $R_{\mathrm{h}}=1$ and $R_{1}=0.1,1$, and 10. Upper and lower panels show linear and logarithmic scale respectively. The image is averaged with GRRT images from $t=14000 M$ to $15000 M$. All averaged images have the same total flux with $0.5 \mathrm{Jy}$ at $230 \mathrm{GHz}$.
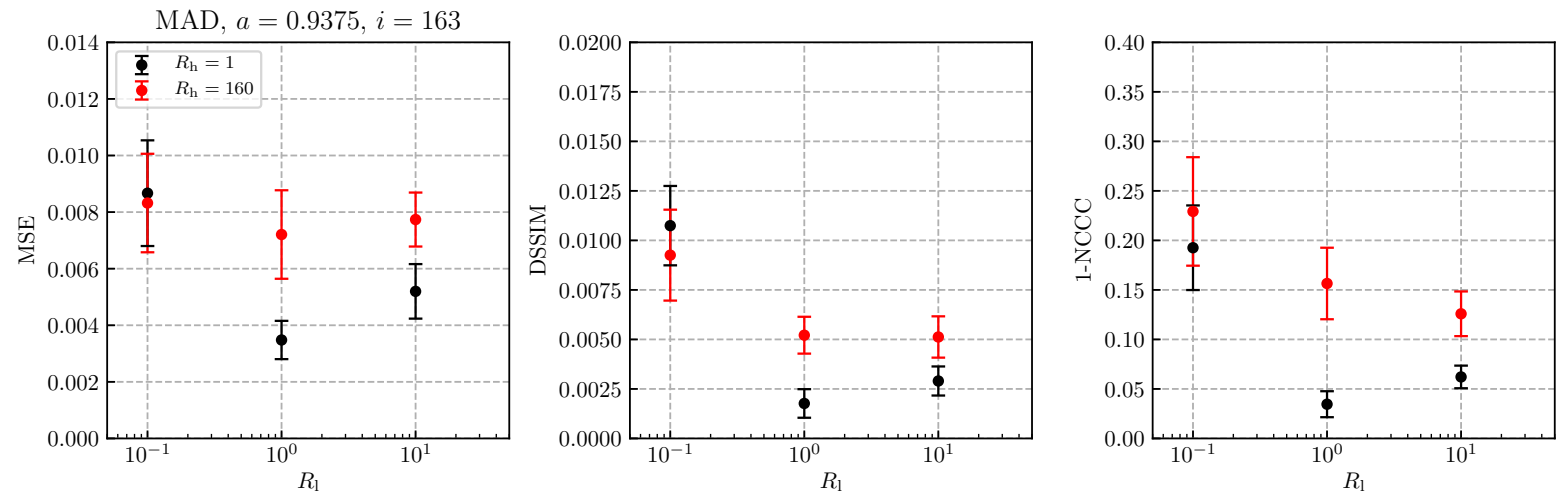

Figure C2. Image-comparison distributions between the turbulent-heating prescription and the parameterised ion-to-electron ratio prescription with different values of $R_{1}$ and a fixed $R_{\mathrm{h}}=1$ (black) and 160 (red) for a black hole with spin $a=0.9375$. Different points indicate the mean value and the shading the standard deviation in the variation. 


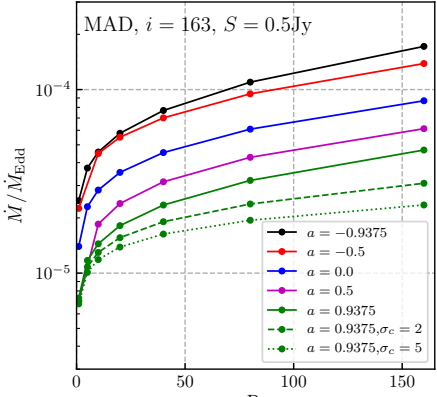

$R_{h}$

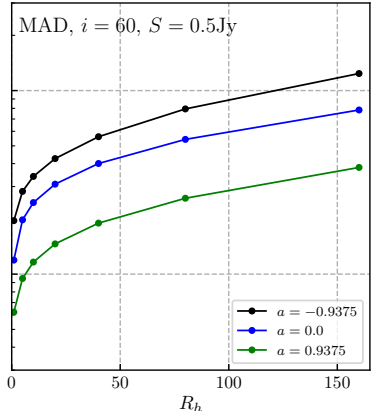

Figure D1. Mass-accretion rate normalised by the Eddington mass-accretion rate for different $R_{\mathrm{h}}$ values when considering different black-hole spins. The mass-accretion rate is computed employing the mass and distance in M87* and by setting the time-averaged flux at $230 \mathrm{GHz}$ to the value of $0.5 \mathrm{Jy}$. 